\title{
Perspectives on Precision Medicine Approaches to NAFLD Diagnosis and Management
}

\author{
Amedeo Lonardo (D) · Juan Pablo Arab (D) - Marco Arrese (D)
}

Received: February 2, 2021 / Accepted: February 26, 2021 / Published online: April 7, 2021

(C) The Author(s) 2021

\begin{abstract}
Precision medicine defines the attempt to identify the most effective approaches for specific subsets of patients based on their genetic background, clinical features, and environmental factors. Nonalcoholic fatty liver disease (NAFLD) encompasses the alcohol-like spectrum of liver disorders (steatosis, steatohepatitis with/without fibrosis, and cirrhosis and hepatocellular carcinoma) in the nonalcoholic patient. Recently, disease renaming to MAFLD [metabolic (dysfunction)-associated fatty liver disease] and positive criteria for diagnosis have been proposed. This review article is specifically devoted to envisaging some clues that may be useful to implementing a precision medicine-oriented approach in
\end{abstract}

\footnotetext{
A. Lonardo $(\bowtie)$

Department of Internal Medicine, Azienda

Ospedaliero-Universitaria, Ospedale Civile di

Baggiovara, 1135 Via Giardini, 41126 Modena, Italy

e-mail: a.lonardo@libero.it

J. P. Arab · M. Arrese

Departamento de Gastroenterología, Escuela de

Medicina, Pontificia Universidad Católica de Chile,

Santiago, Chile

J. P. Arab · M. Arrese

Departamento de Biología Celular y Molecular,

Centro de Envejecimiento y Regeneración (CARE),

Facultad de Ciencias Biológicas, Pontificia

Universidad Católica de Chile, Santiago, Chile
}

research and clinical practice. To this end, we focus on how sex and reproductive status, genetics, intestinal microbiota diversity, endocrine and metabolic status, as well as physical activity may interact in determining NAFLD/ MAFLD heterogeneity. All these factors should be considered in the individual patient with the aim of implementing an individualized therapeutic plan. The impact of considering NAFLD heterogeneity on the development of targeted therapies for NAFLD subgroups is also extensively discussed.

Keywords: Cirrhosis; Fibrosis; Metabolic syndrome; NAFLD; NASH; Nonalcoholic fatty liver disease; Nonalcoholic steatohepatitis; Steatosis 
Key Summary Points

Precision medicine tries to identify the most effective approaches for specific subsets of patients based on their genetic background, clinical features, and environmental factors.

Nonalcoholic fatty liver disease (NAFLD), now renamed metabolic (dysfunction)associated fatty liver disease (MAFLD), encompasses the heterogenous alcohollike spectrum of liver disorders in the nonalcoholic.

This review article aims at envisaging useful clues to implementing a precision medicine-oriented approach in NAFLD/ MAFLD research and clinical practice.

We discuss how sex and reproductive status, genetics, intestinal microbiota diversity, endocrine and metabolic health, as well as physical activity may interact in determining NAFLD/MAFLD heterogeneity.

We also attempt to envision a tailored management approach based on each of the factors outlined above.

\section{DIGITAL FEATURES}

This article is published with digital features, including a summary slide, to facilitate understanding of the article. To view digital features for this article, go to https://doi.org/10.6084/ m9.figshare.14113481.

\section{INTRODUCTION}

Personalized medicine is part of the "4P Medicine" approach, which intends to advance clinical care to a preventive, predictive, personalized, and participatory model $[1,2]$. The expression "personalized medicine" is often used interchangeably with "precision medicine" which, in its wider definition, focuses on identifying the most effective approaches for specific subsets of patients based on their genetic background, environmental factors, and lifestyle features including their social and environmental profile. Precision medicine approaches promote a deeper understanding of the disease process in any individual patient. This is believed to better predict clinical outcomes and maximize the therapeutic benefits of a given treatment while keeping adverse effects to a minimum [3]. To this end, a more accurate and refined characterization and stratification of disease is needed while assessing individual patient pathologies by applying multiple molecular tools (e.g., omics, genetic testing, microbiome assessment) and advanced imaging techniques as well as a detailed evaluation of the patient's clinical features [4]. Indeed, it has recently been proposed that precision medicine paradigms should also be applied to the hepatology arena following behind other medical disciplines including oncology and genetic diseases [5-8].

NAFLD, the acronym of nonalcoholic fatty liver disease, is a definition originally proposed by Shaffner and Thaler in the mid 1980s based on an earlier definition coined by Ludwig et al.: nonalcoholic steatohepatitis (NASH) [9]. NAFLD, of which NASH is the most rapidly progressive histological variant, is an umbrella definition which includes the gamut of fatty liver syndromes spanning simple steatosis through NAFLD-hepatocellular carcinoma (HCC), which usually occur via NASH and NAFLD-related cirrhosis [10]. As a universally widespread condition and owing to a large variety of hepatic and extrahepatic manifestations, complications, and concurrent conditions, NAFLD has been the subject of intensive research over the last 40 years [9, 11-13]. Over time, the adjective "nonalcoholic" [9], however, has been much criticized for a variety of reasons, notably including the fact that it identifies something for which it is not rather than something for which it is [14]. Following pioneer attempts, several authors have proposed to rename NAFLD, the most recent of such proposals resulting in the definition of this disease 
as MAFLD, i.e., metabolic (dysfunction)-associated fatty liver disease, which aims at emphasizing the strong connections of NAFLD with diabetes and obesity in most individuals $[15,16]$. Although the initiative to rename NAFLD to MAFLD has generally been welcomed by the scientific community [17-19], the suggestion to change the name has been felt to contain some elements of prematurity and a debate is presently ongoing $[20,21]$.

Recent advances in our knowledge of NAFLD genetics and in systems biology have fueled the potential application of precision medicine approaches to this disease $[5,6,22]$. In the present review article, we aim at providing a critical overview of selected research topics that relate to a more precise sub-phenotyping of patients with NAFLD based on sex and reproductive status, genetics, intestinal microbiota diversity, endocrine and metabolic status, as well as the degree of physical activity. Proper consideration of these factors may lead to more precise diagnostic approaches that, in turn, could be translated into improvements in efficacy and safety of both conventional and experimental therapeutic strategies.

\section{RATIONALE AND METHODS}

The historical backdrop of the difficulties in identifying the best nomenclature to designate NAFLD (reviewed in [14]) reflects itself in disappointing therapeutic results. Decades of intensive research have indeed led to an improved understanding of disease epidemiology and pathogenesis $[9,23-25]$ but this has not yet translated into success in identifying effective drug treatments. In fact, a significant portion of investigational drugs have failed in showing efficacy even in late phase randomized controlled trials (RCTs) $[26,27]$. While myriads of explanations may, in principle, account for this therapeutic failure, it should constantly be kept in mind that the pathogenesis of disease is multilayered and extraordinarily composite $[28,29]$, which may explain the variability, in the individual patient, of NAFLD clinical course and outcomes, from a benign to a progressive and deadly disease, and from hepatic to extrahepatic manifestations and complications.

The recent proposal to rename NAFLD to MAFLD [15, 16] has fueled expectations that this change in nomenclature may help, or at least contribute, to better identifying more homogeneous patient subgroups to be included in future clinical trials [15]. However, while consensus is growing, evidence remains lacking. In the meanwhile, we consider that patients with NAFLD included in clinical trials should be evaluated with the most in-depth approach possible aimed at trying to define more homogenous subgroups of patients that may better respond to novel investigational drugs.

Epistemology defines the logical foundations of scientific thinking. From the epistemological point of view, there are two main approaches: the inductive theory and the deductive theory [30]. The inductive method is based on the systematic observation and classification of findings. These are critically examined and, based on whether they are consistent/inconsistent with the interpretative hypothesis, they will lead to either accepting or rejecting the hypothesis [30]. Stated in short, this approach implies that "observing and thinking" (observatio and ratio in Latin) is sufficient to reach a medical diagnosis. In contrast, the deductive method is based on a hypothesis-driven approach which will inform the diagnostic flowchart aiming to either prove or reject the originary hypothesis. In short, the deductive method may be likened to an electric torch that the clinician directs in order to shed light on the most obscure spots she/he wants to focus on [30]. These general principles of medical epistemology may also be applied to NAFLD, an arena in which individual variability in response to metabolic stress and in liver tissue repair are acknowledged determinants of heterogeneity in disease pathobiology and susceptibility to hepatic fibrosis due to the multiphasic pathogenesis of NAFLD/NASH $[28,31,32]$. Severity of hepatic fibrosis, in its turn, is a key player in the course of hepatic and extrahepatic diseases given that liver-related events predominantly affect patients with NAFLD-cirrhosis, whereas those with bridging fibrosis will mainly develop non-hepatic cancers 
and vascular events $[33,34]$. This implies that information obtained from liver biopsy is key in predicting the outcome of the individual patient. However, given the limitations of liver biopsy, non-invasive techniques may also fruitfully be applied to this end [35]. Therefore, what we need is an electric torch systematically exploring (either invasively or non-invasively) all the "dark spots" of NAFLD variability.

Similar to other complex disorders, such as type 2 diabetes (T2D), arterial hypertension, and cancer, our therapeutic approach to NAFLD should best be strongly personalized and based on the individual features of disease and on its expected outcomes (i.e., the natural history of disease).

In order to prioritize the vast biomedical literature published on this topic, in January 2021, PubMed was consulted using the following search terms: "NAFLD", "nonalcoholic fatty liver disease", "NASH", "steatosis", "fatty liver", "precision medicine", "steatohepatitis", "metabolic syndrome", "MAFLD". Guidelines, original articles, review articles, editorials, and their reference lists were considered. There were no language restrictions nor specific exclusion criteria. Inclusion of proposed articles was based on unanimous agreement among the authors. This article is based on previously conducted studies and does not contain any new studies with human participants or animals performed by any of the authors.

\section{COMPLIANCE WITH ETHICS GUIDELINES}

This article is based on previously conducted studies and does not contain any new studies with human participants or animals performed by any of the authors.

\section{DIAGNOSIS}

Further to standard assessment, which includes the use of biomarkers and imaging techniques as the initial step [36-39], the NAFLD diagnostic process should include a more accurate definition of a series of parameters mirroring the extent and severity of hepatic and extrahepatic involvement $[40,41]$. The recently proposed "LDE system" $[42,43]$ is an example of a possible synthetic descriptor of variables related to the liver $(\mathrm{L})$, to the determinants of disease specific to the individual patient (D), and to the extension of extrahepatic involvement (E) that is typical of a systemic disorder such as NAFLD [44].

L-Estimation of liver disease severity in patients with NAFLD is currently focused on the assessment of the presence and degree of liver fibrosis as this feature is the main determinant of mortality among these patients and accurately predicts both all-cause and disease-specific mortality in patients with NAFLD $[24,45]$. A large variety of biomarkers, algorithms, and imaging techniques collectively grouped under the heading of "non-invasive diagnostic tools" are available to estimate the degree of liver fibrosis in NAFLD [46]. Table 1 summarizes advantages and limitations of each approach that have been extensively reviewed elsewhere [35]. In addition, recent studies have pinpointed that thresholds of non-invasive fibrosis scores, such as Hepamet, FIB-4, and NFS, can be modified according to the ethnicity of patients with NAFLD with the aim of reaching maximal diagnostic accuracy [47]. In addition, liver fibrosis biomarkers such as aspartate aminotransferase (AST) to alanine aminotransferase (ALT) ratio (ARR), AST to platelet ratio index (APRI), Fibrosis-4 (FiB-4), Forns index, NAFLD fibrosis score (NFS), BARD (body mass index (BMI), AAR, diabetes) score, and Hepamet fibrosis score (HFS) can rule out advanced fibrosis and are positively correlated with cardiovascular risk (CVR) scores in patients with NAFLD [48]. The role of the HFS should best be further explored in prospective studies as well as when combined with other diagnostic methods [49].

D-Assessment of disease determinants in individual patients is not routinely performed in clinical practice nor in RCTs despite the fact that a variety of pathogenic risk factors are well characterized. In our view, all the following features are important modifiers that are worth registering in the individual patient and should be taken into consideration in therapeutic 


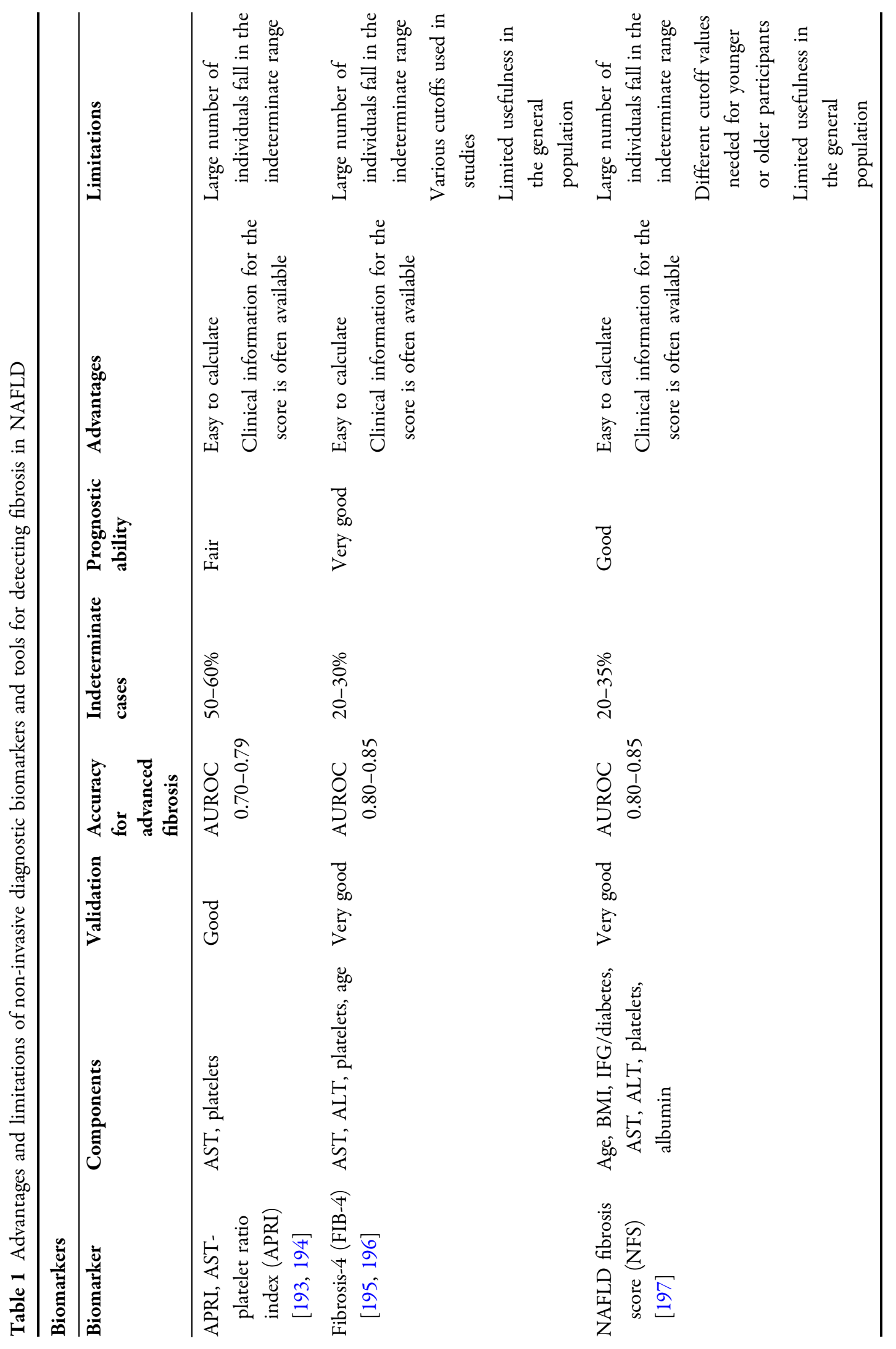




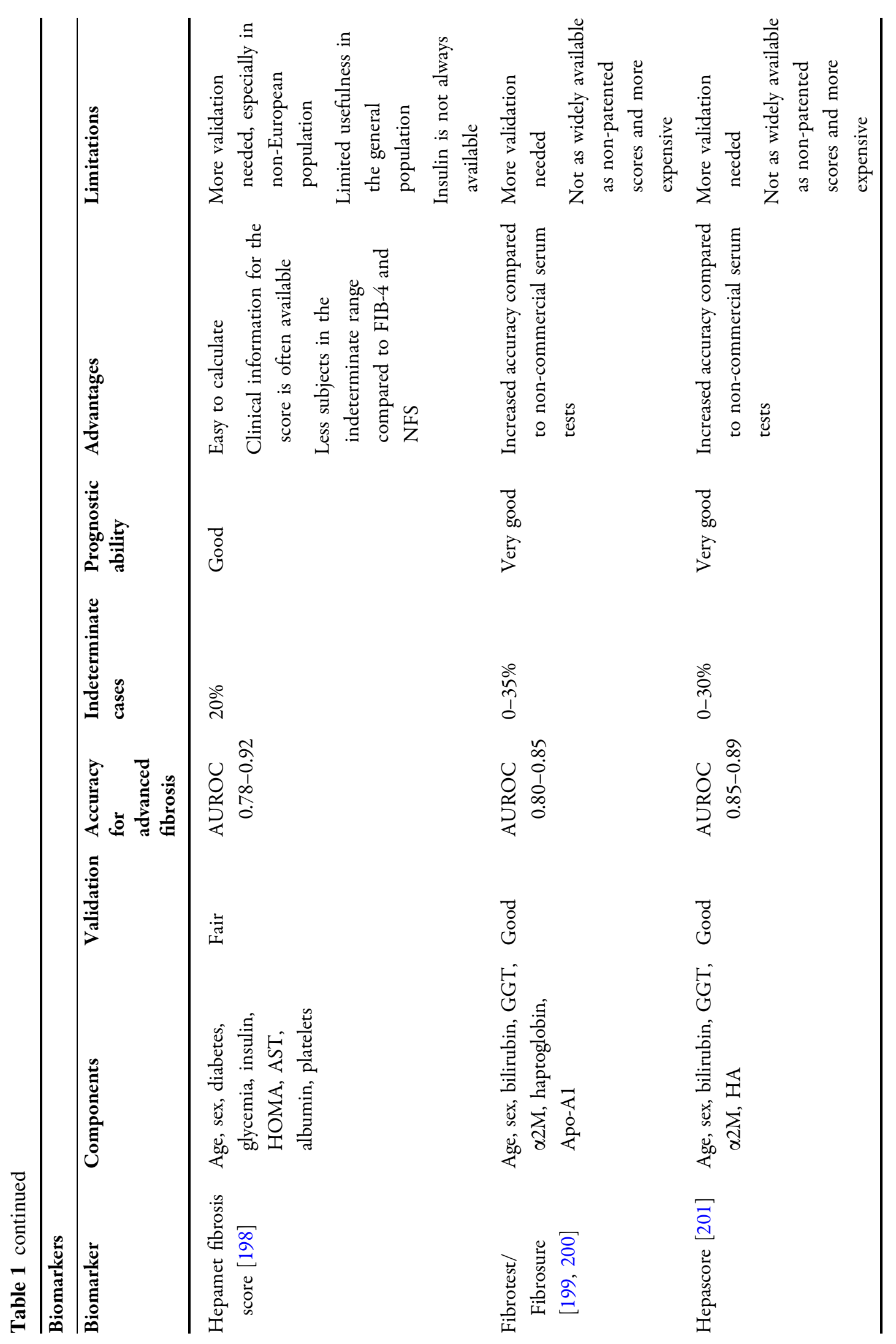




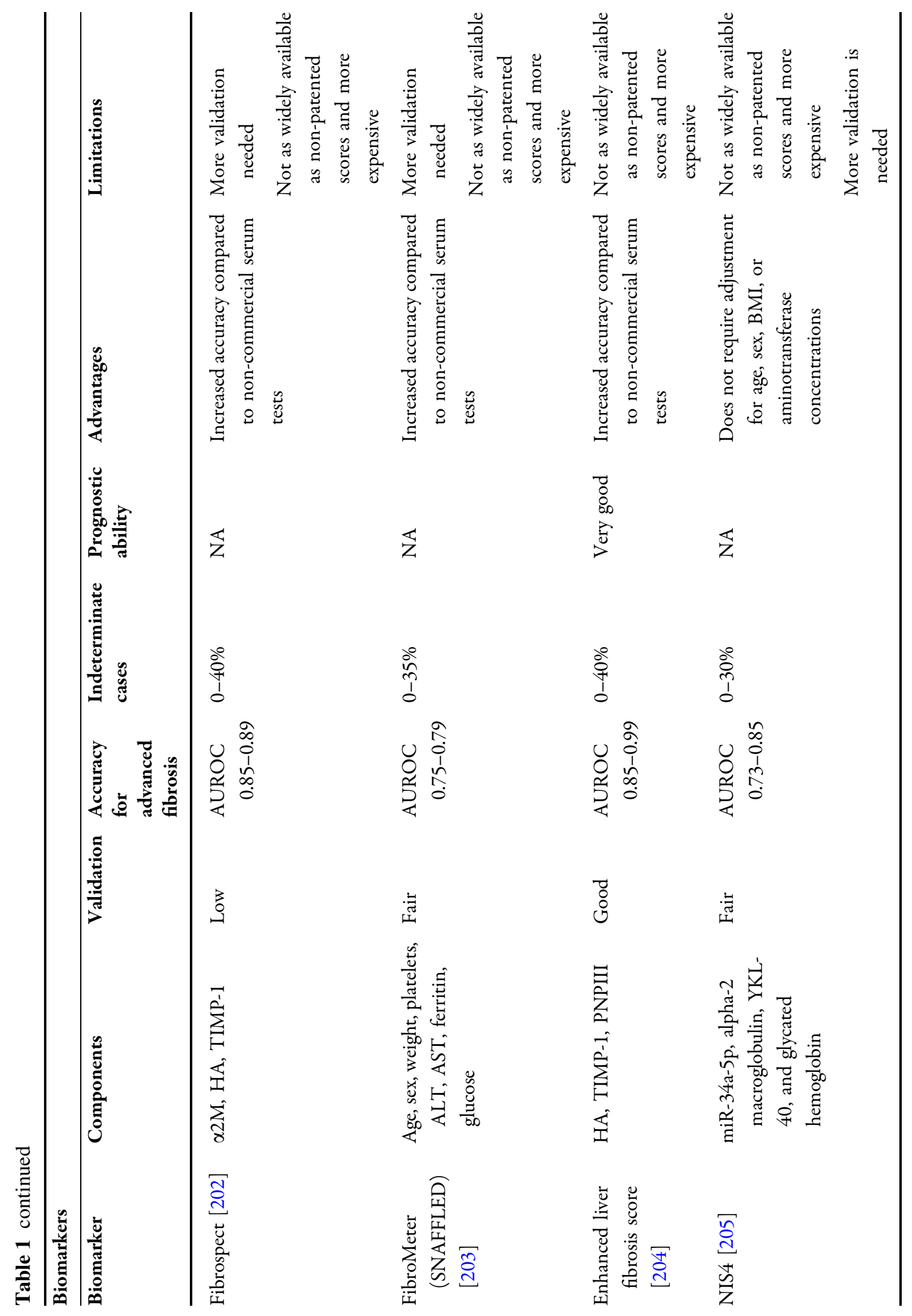




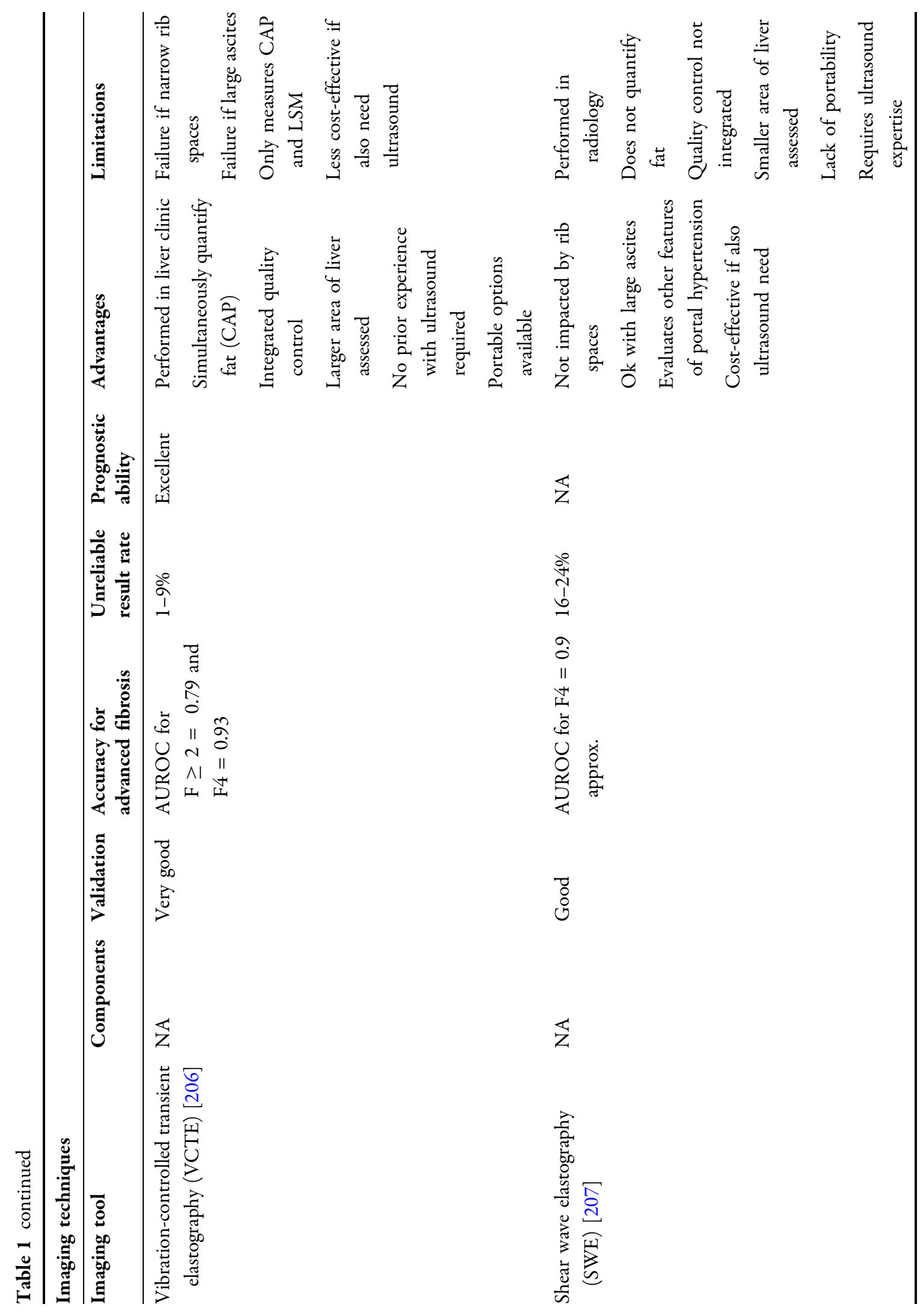




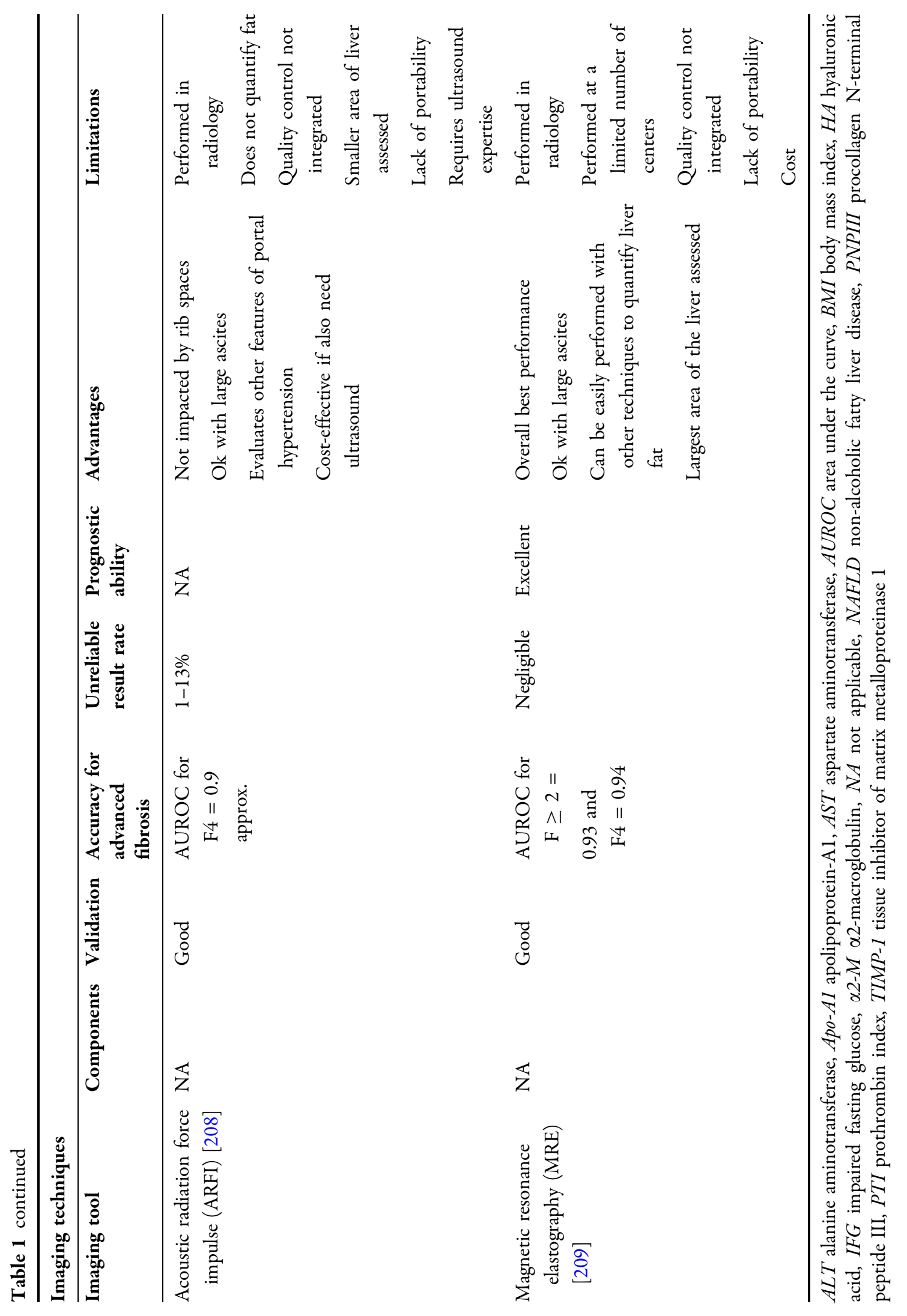


studies: (i) sex and reproductive status, (ii) genetics, (iii) intestinal microbiota, (iv) endocrine assessment, (v) metabolic assessment, and (vi) physical activity. Existing data on the role of these factors in NAFLD are summarized in "Sex, Gender, and Reproductive Status".

$\mathrm{E}$-The extent of extrahepatic manifestations and complication of NAFLD exhibits an everenlarging spectrum of conditions spanning, for example, T2D to chronic kidney disease and colorectal cancer, just to name a few. This spectrum has extensively been reviewed elsewhere [13]. For clinical purposes, it is important to categorize each patient as being affected by metabolic derangements [50], cardiovascular disease [51], and/or non-hepatic cancer [52]. This categorization promises to contribute to a more accurate risk stratification, personalized follow-up protocols, and full consideration of polypharmacy.

\section{Sex, Gender, and Reproductive Status}

Sex and gender are major modifiers of a series of common conditions pertaining to the domain of internal medicine including heart, lung, and kidney disease [53]. Sex medicine is the first step of personalized medicine in as much as, for example, sex is one of the major determinants of drug dose level, responses, and adverse reactions [54]. NAFLD also has definite sexual dimorphic features [55]. A recent meta-analytic study found that women were more protected from the risk of developing bland steatosis than men, while being at an increased risk of fibrosis progression than men [56].

Gender defines a social attribute as opposed to sex that is a biological feature [53]. Little is known regarding the role of gender in the development and progression of NAFLD. However, it is conceivable to postulate that gender attributes, such as drinking alcohol, may contribute to the increased risk of developing HCC in men with NAFLD $[57,58]$.

Reproductive status is also a strong determinant of the risk of development and progression of NAFLD given that postmenopausal NAFLD tends to mimic epidemiological features such as those seen in men [59-61].
On this background, experts have recommended that sex and reproductive status should receive adequate consideration when planning NAFLD research $[59,62,63]$.

\section{Genetics}

The risk of developing NAFLD and related complications has been clearly related to genetic factors. Genome-wide association studies (GWAS) have consistently shown associations between a set of genetic variants and NAFLD development and severity [64]. The main genes uncovered by these studies are PNPLA3, TM6SF2, MBOAT7, GCKR, and HSD17B13 (Table 2), with the PNLA3 I148 variant (rs738409) being the most extensively characterized [65]. Of note, having this variant carries an approximately doubled risk of developing NAFLD and, of concern, a tripled risk of NASH and HCC per allele $[6,65]$. Interestingly, while the PNPLA3 gene 148Met allele and the TM6SF2 gene 167Lys allele are associated with an increased risk of steatosis development and progression (to NASH and cirrhosis) they seemingly protect from cardiovascular disease $[66,67]$. This feature might be leveraged to conduct a stricter hepatological follow-up in carriers of these variants as opposed to "metabolic NAFLD" wherein cardiovascular assessment is strongly recommended [37, 64]. Of note, genetic variants are not present in all individuals with NAFLD and, based on current understanding, as pointed out by some authors it is uncertain whether "metabolic NAFLD" and "genetic NAFLD" are entirely equivalent $[68,69]$. Although routine genotyping of patients is not yet recommended, the use of polygenic risk scores (PRS), which are based on the sum of all independent risks conferred by carrying a single nucleotide polymorphism (SNP), is being investigated as a useful tool to identify patients at risk of developing more severe disease [5] or complications, such as HCC [70]. 


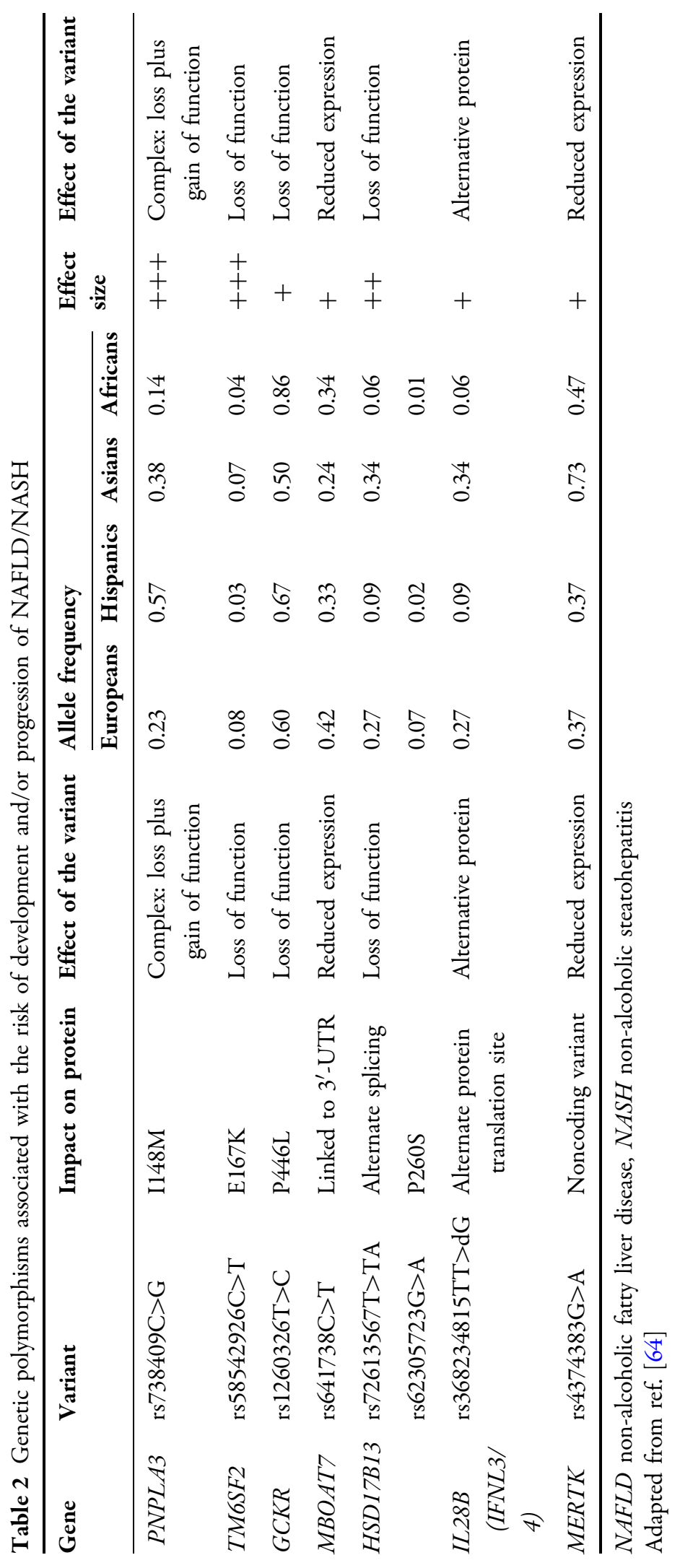




\section{Intestinal Microbiota}

Gut-liver axis signaling pathways, such as microbiota-related mechanisms (i.e., dysbiosis, production of endogenous ethanol, and choline deficiency) play a major role in the pathogenesis of NAFLD and NASH [71]. This may occur either directly or indirectly (i.e., via altered metabolism of bile acids) and is deemed to be a key element of personalized medicine $[44,72,73]$. Indeed, lifestyle changes are closely linked with modulation of the intestinal microbiota, which may potentially account for the inter-individual variability of patients with NAFLD [72]. Both experimental and clinical data show the relevance of diet-microbiota interaction in determining metabolic abnormalities. For example, germ-free mice exhibit less pronounced steatosis and do not become obese when fed a western-type diet [74]. Obesity is strongly associated with altered gut microbiota [72], and a diet rich in vegetables and fiber (i.e., rural African diet) is associated with a high Bacteroidetes to Firmicutes ratio [75], which is maintained even in the presence of a high-fat diet $[72,76]$.

The highly lipogenic sugar fructose, deemed to be a major risk factor of the development of NAFLD [77, 78], is a key player in intestinal dysbiosis, increased gut permeability, portal blood endotoxemia, and again in determining an altered Bacteroidetes to Firmicutes ratio, which characterizes NASH. Finally, progress in harnessing intestinal microbiota as a potential diagnostic tool in NAFLD/NASH as well as NASH-related HCC is being made [71, 79]. Data on the existence of distinct microbiota signatures associated with significant fibrosis $[80,81]$ is of particular interest in this regard. Thus, it is likely that in the near future simplified assessment of intestinal microbiota composition may help in better characterizing patients with NAFLD/MAFLD.

\section{Endocrine Assessment}

In clinical practice and in RCTs, the amplitude of the diagnostic protocol aimed at ruling out competing causes of liver disease remains poorly defined in patients with NAFLD. However, "endocrine-related NAFLD and NASH" forms do exist and have been discussed elsewhere [82, 83]. Based on the finding that hypothyroidism-induced NAFLD (HIN) is commonly seen in clinical practice and on the high prevalence of hypothyroidism in general population studies, it has been proposed that thyroid status should be assessed through the routine evaluation of thyroid-stimulating hormone (TSH) $[42,84-86]$.

So distinctive is the pathogenesis of liver disease in these cases and given the potential for total reversibility of disease with thyroid hormone replacement therapy that HIN has been proposed as a distinct disease entity $[87,88]$.

In the clinic, clues from signs and symptoms of androgen excess and ovarian dysfunction (e.g., menstrual irregularities) may suggest the presence of polycystic ovary syndrome (PCOS) [89], a condition which is deemed to be strongly associated with progressive forms of NAFLD $[90,91]$.

Other well-defined forms of NAFLD/NASH secondary to specific endocrine disorders include growth hormone $(\mathrm{GH})$ deficiency $[92,93]$, which is deemed to mirror the failure of low GH levels to inhibit hepatic de novo lipogenesis [94]. Phenotypically, in adults, GH deficiency exhibits NAFLD, NASH (whose histology will significantly improve following administration of GH to GH-deficient adults), and NASH-cirrhosis associated with visceral obesity, dyslipidemia, and premature atherosclerosis [95].

Data regarding glucocorticoid excess appear to be less robust: on the basis of computerized tomography findings, Rockall et al. found steatosis in $20 \%$ of patients with Cushing's syndrome [96]. It has been speculated that an excess of cortisol, by inhibiting systemic lowgrade chronic inflammation mainly mediated by interleukin-6, could account for this protective effect from developing NAFLD [97]. Auer et al. found that NAFLD, assessed with a surrogate index, fatty liver index (FLI), in 33 patients with biochemically controlled Cushing's disease was not more common than that found among 79 individuals with non-functioning pituitary adenomas [98]. 
In conclusion, while screening for TSH alterations seems justified in the standard evaluation of NAFLD given the common occurrence of HIN, PCOS may be suggested by clinical findings. Finally, evaluation of GH deficiency and hyperadrenalism should be reserved for selected cases based on clinical suspicion.

\section{Cardio-Metabolic Assessment}

A first-level assessment of common anthropometric indices (such as BMI and waist circumference) should routinely be evaluated to differentiate the various subtypes of NAFLD, such as NAFLD in the obese (high BMI), in the visceral obese (high waist circumference with/ without high BMI), or in the non-obese (normal BMI and waist circumference). Also, blood pressure should be recorded together with the use of antihypertensives, given that high blood pressure is a risk factor for the progression of liver fibrosis [99].

The dangerous association of impaired glucose disposal and NAFLD is widely acknowledged [100, 101]. For example, a recent study found that fatty liver, when associated with dysglycemia, had the potential to progress to chronic kidney disease (CKD) as defined by reduced estimated glomerular filtration rate (eGFR) [102]. Similar to obesity, the various grades of normal, impaired, and frankly altered gluco-tolerance should be evaluated and recorded with appropriate static and dynamic testing [103].

The diagnosis of MAFLD, as opposed to NAFLD, may partially obviate the need to specify if the patient has diabesity or not, but whether the risk of progression is the same among those who have diabetes and among those who are obese remains to be proven.

Types and severity of lipid metabolism disorders should be registered [104]. A study has reported that hypertriglyceridemia was a risk factor for cirrhosis in overweight Swedish individuals [105], a finding that may suggest unrecognized T2D as the culprit. It has also been reported that an atherogenic lipoprotein profile is related to NAFLD severity and progression to cirrhosis [106]. In addition, the pathogenic role of cholesterol in the pathogenesis of NASH is well characterized [107, 108] and accumulating epidemiological data support the beneficial effects of statins in this setting [109-111]; however, evidence is observational and well-conducted RCTs remain to be performed.

It is widely acknowledged that NAFLD is closely associated with an increased risk of conditions of the cardiovascular system. These comprise both anatomic abnormalities and perturbed heart function [112, 113], notably including an increased risk of atrial fibrillation [112]. The venous system may also be involved, and it has indeed been reported that patients with NAFLD are prone to the risk of venous thromboembolism [112].

Finally, although in the absence of clear-cut data suggesting that venesection cures NAFLD/ NASH [114] and given that in NAFLD hepatic iron overload is strictly associated with insulin sensitivity [115], it may be logical to record the levels of ferritin and to further investigate all patients with percent saturation of transferring above the cutoff limits [116].

\section{Physical Activity}

It has long been known that, compared to nonNAFLD controls, subjects with NAFLD have significantly lower levels of physical activity [117]. Most patients with NAFLD exhibit the socalled triple-hit behavioral phenotype consisting in (i) sedentary behavior, (ii) low physical activity, and (iii) poor diet [118]. Short-term benefits of physical activity in NAFLD are well demonstrated [119] and it is likely that physically active individuals with NAFLD, similar to patients with $\mathrm{T} 2 \mathrm{D}$, may be protected from developing complications of their disease leading to a reduced healthcare financial burden [120]. On these grounds, it seems relevant that patients with NAFLD should always be classified along a semi-quantitative scale of sedentary behavior defined as time spent sitting or lying with low energy expenditure (e.g., assessed with TV watching time) and physical activity (along a scale of intensity and time spent on exercising per week). That information, in addition to 
proper evaluation of cardiovascular status, would help to prescribe an adequate and progressive exercise program ensuring compliance. Interestingly, some studies conducted in animal models as well as in human twins point to the existence of significant inter-individual differences in the response to a given exercise program dose with regard to achievement of cardio-respiratory fitness and modification of cardio-metabolic features [121, 122], which opens the possibility of personalized exercise program prescription.

\section{TAILORING NAFLD MANAGEMENT TO INDIVIDUAL PATIENTS}

Principles of NAFLD management have been outlined in several clinical guidelines or position papers released and endorsed by either scientific societies or expert panels [36-38]. All these documents agree that lifestyle modifications remain the cornerstone of NAFLD management and are indicated in all patients [123]. Data suggest that a Mediterranean diet is one of the nutrition styles to be recommended, associated with caloric restriction oriented to achieve a $7-10 \%$ loss of basal body weight [118]. Dieting should be combined with exercise according to current recommendations issued by the American College of Sports Medicine (ACSM) and World Health Organization (WHO) taking into account basal cardiovascular risk as well as comorbidities [119, 124]. Currently, there are no approved specific medications for NAFLD/NASH but several agents are under investigation in phase 3 trials and may offer new treatment alternatives in the near future $[125,126]$.

Precision medicine approaches in NAFLD treatment are still in their infancy $[5,6,22]$. Indeed, more information needs to be gathered to generate tailored therapeutic plans to the different and heterogenous patient categories grouped under the acronym NAFLD. In the following paragraphs, we attempt to envision a targeted approach based on the factors outlined above.

\section{Sex and Reproductive Status}

Human metabolism is different between sexes and is critically influenced by reproductive status. Thus, these variables could be of importance when planning lifestyle interventions in patients with NAFLD [127]. Of note, emerging data from experimental models also suggest sex differences in response to dietary restrictions [128]. Therefore, while female mice respond better to caloric restriction in terms of longevity [129], non-human primates show a male-specific reduction in body weight and increase in insulin sensitivity when subjected to this dietary limitation [130]. However, information about this matter in humans is scarce because intervention studies do not routinely stratify according to sex [131]. Some studies suggest that, in general, men tend to lose more weight and exhibit more metabolic benefits than women when undergoing lifestyle intervention programs [132]. Interestingly, in one of the most cited studies assessing lifestyle interventions in NAFLD [133], men showed a greater histological improvement than women after weight loss. In this study, male sex was one of the factors predicting beneficial histological response following a relatively modest weight loss (between 7\% and 10\%), while in women a more substantial weight loss (more than 10\%) was required to achieve a significant histological improvement [133]. Therefore, on the basis of available data, women may require additional support to achieve their goals after introduction of lifestyle changes.

With regard to reproductive status, studies on the effect of lifestyle modifications on NAFLD specifically in postmenopausal women are limited [60, 134]. A recent metanalysis of published data suggests that weight loss interventions may not need to be tailored to women's menopausal status [135].

\section{Genetics}

The robust associations of NAFLD development and progression with specific gene variants (summarized in Table 2) may allow the utilization of genotyping of any given patient to aid 
diagnosis or predict disease trajectories, thus allowing for better prognostication [65]. Moreover, once precise pathophysiology is deciphered, gene-specific therapy would be possible [136]. In spite of a plethora of information regarding the influence of genetic variants on disease severity, cardiovascular risk, and risk of developing cirrhosis and HCC, translation into clinical practice is still pending because of lack of data supporting cost-effectiveness of routine genetic testing in patients with NAFLD. Of all genetic variants identified as relevant for NAFLD development and progression (Table 2), PNPLA3 is the most studied and the p.I148M variant of the gene encoding this protein is consistently associated with a more severe disease and an increased risk of HCC. It has also been associated with both liver-related and allcause mortality in a general US population [65]. Moreover, it has been shown that patients carrying the p.I148M variant also exhibit an antiatherogenic lipid profile and a better response to lifestyle modifications and bariatric surgery [137-139]. This information may help to implement specific measures, such as personalized cardiovascular risk management, lifestyle intervention, and tailored HCC screening programs in patients with NAFLD. Additionally, as discussed above, genetic information may be used through the use of PRS, which reflect the risk accumulation determined by harboring multiple SNPs and may assist in disease prediction. In a recent paper, Bianco et al. showed that the use of PRS, in which PNPLA3, TM6SF2, $G C K R$, and MBOAT7 were combined, may improve HCC risk stratification in NAFLD [70]. Thus, in the near future, the challenge is to generate data showing whether decision-making based on patients' genetic information is cost-effective or not.

From the therapeutic standpoint, data from experimental models have shown that it is possible to improve all histological features of NAFLD in vivo by silencing PNPLA3 with livertargeted antisense oligonucleotides [140]. Therefore, in future, specific therapy may be amenable for those individuals carrying the NAFLD p.I148M variant, which would be indicated only in "genetic NAFLD" as opposed to "metabolic" disease [68].

\section{Manipulation of Intestinal Microbiota}

The growing amount of data on intestinal microbiota in patients with NAFLD have opened the possibility to develop microbiomebased approaches to manage the disease $[71,141]$. As discussed above ("Intestinal Microbiota"), "microbiome signatures" can be used for personalizing NAFLD diagnosis and staging. Moreover, these could also be useful for selecting patients to submit to therapeutic fecal microbiota transplantation (FMT) as well as biomarkers of response to therapy [142]. This is relevant considering that not all patients have either gut dysbiosis or increased intestinal permeability [143] and that it is very likely that certain subsets of patients will respond better to microbiota-targeted therapies than others. Therefore, microbiome-based biomarkers could be applied for developing more targeted management approaches such as dietary interventions, manipulation of gut microbiota through the use of probiotics, prebiotics, and/or synbiotics as well as FMT or bacteriophage therapy $[71,142]$. To date, studies on the use of probiotics and synbiotics in NAFLD suggest some benefit in terms of amelioration of hepatic inflammation, steatosis, and eventually liver fibrosis [144], but data heterogeneity is significant and well-designed RCTs are lacking. Indeed, one source of heterogeneity is the diverse microbiota composition and differences in treatment regimens that affect bacterial engraftment. Interestingly, this seems to be predicted by individual baseline gut microbiota [145].

\section{Endocrine Considerations}

\section{Thyroid Disease and Thyromimetics}

As previously argued, hypothyroidism is commonly found among patients with NAFLD [84]. It is predicted that thyroid hormone replacement could fully reverse the disease or at least improve disease progression leading to better outcomes [88]. Of note, it has been proposed that HIN should be considered as a potentially curable and distinct disease entity [87]. In any case, routine evaluation of the presence of 
thyroid disease should be carried out in any patient with NAFLD and treatment instituted when indicated. Levothyroxine administration has been shown to reduce serum aminotransferases and hepatic fat content in either euthyroid or subclinical hypothyroid patients with NAFLD [146, 147]. Long-term studies assessing liver disease progression and other outcomes are lacking. One interesting application of the thyroid-liver axis in NAFLD is the potential therapeutic use of liver-specific thyromimetics [88]. These drugs are agonists of the hepatic thyroid hormone receptor isoform beta (THR- $\beta$ ) and are devoid of systemic effects. Two drugs are under study, VK2879 and MGL-3196, also named resmetirom, with the latter showing promising results in terms of liver fat reduction in a phase 2 trial $[148,149]$. Results of phase 3 clinical trials are eagerly awaited. Considering their mechanism of action, it is likely that thyromimetics may be more effective in early stages of the disease [148]. Thus, once proven effective when available, this may be a consideration for personalized prescription.

\section{Hormonal Therapy in NAFLD Associated with PCOS, Hypogonadism, GH Deficiency, and Other Endocrine Disorders}

Patients with NAFLD and concurrent endocrinopathies should be evaluated by a specialist in order to receive optimal treatment. As reported above, PCOS is independently associated with more severe liver disease, including advanced fibrosis and cirrhosis [91]. This implies that it is important to screen for PCOS in all women referred for NAFLD who have medical histories and clinical findings compatible with PCOS given that these patients require appropriate hepatological follow-up and intensive management. In particular, the use of metformin has proven effective in decreasing insulin resistance in these patients, although its efficacy on NAFLD histology remains unproven.

Male patients with hypogonadism and NAFLD could possibly benefit from testosterone administration [150, 151]. However, the longterm safety profile of this therapy, including cardiovascular outcomes, remains unclear.

GH replacement therapy is recommended in cases of symptomatic hormone deficiency, which may impact insulin sensitivity, visceral fat, and dyslipidemia [152]. However, data on its effect on human NAFLD are limited [93]. The same holds true in the case of GH excess where the impact of therapy on NAFLD remains to be evaluated [93].

\section{Cardiometabolic Considerations}

As previously pinpointed, cardiovascular disease and $\mathrm{T} 2 \mathrm{D}$ are among major comorbidities of NAFLD. Indeed, when planning a tailored treatment scheme, these two aspects should be carefully assessed and best tackled in the context of a multidisciplinary team. Although evidence is scarce, a holistic multidisciplinary management seems to be superior to standard approaches by determining better improvements to liver and cardio-metabolic health [153].

\section{Cardiovascular Disease}

The field of precision cardiology is rapidly developing [154, 155]. Since patients with NAFLD may suffer from coronary artery disease and other cardiovascular conditions (i.e., arterial hypertension, left ventricular hypertrophy, certain arrhythmias, and valve calcification [156]), modern concepts of precision cardiology could be applied to gather data regarding risks of individual patients, thereby boosting the capacity to diagnose and estimate prognosis while planning future treatments $[4,157]$. Coronary heart disease and arterial hypertension are indeed conditions that may be susceptible to a more precision-oriented approach beyond current guidelines [4]. Combined approaches using modern cardiovascular risk assessment concepts, novel biomarkers, specific genotyping of NAFLD cases, advanced imaging, and novel biomarkers may serve to stratify patients with NAFLD in terms of cardiovascular risk and allow for more personalized treatment decisions. In this regard, the proper use of statins in patients with NAFLD to prevent the development and complications of atherosclerosis in patients at a high risk is very relevant considering that these drugs are often underprescribed [156]. Of note, in the near future a precision medicine 
approach to lipid-lowering therapy is also envisioned [158].

Both venous thromboembolism and atrial fibrillation are amenable to anticoagulation therapy. In the absence of any standardized indications, the use of direct oral anticoagulants should be personalized. Personal history (high risk vs. low risk of thromboembolic complications), stage of disease (cirrhotic vs. non-cirrhotic), and individual risk of bleeding complications should be carefully balanced in the individual patient [112].

\section{Type 2 Diabetes}

As mentioned above, on the basis of the common association of both conditions, patients with NAFLD should be screened for T2D [100]. Additionally, given that NAFLD is a definite risk factor for incident T2D [159], a preventive approach is justified in these patients [160]. Importantly, significant liver fibrosis is an independent risk factor for T2D appearance with a hazard ratio of 2.95 (95\% CI 1.19-7.31) [161]. On the basis of their higher risk of cirrhosis, patients with concurrent NAFLD and T2D should receive individualized management of T2D that seeks optimal metabolic control according to current guidelines [100, 162]. Although metformin does not seem able to improve histological endpoints in NAFLD, recent studies have shown that patients with diabetes and NASH-related cirrhosis treated with metformin do exhibit higher rates of survival and lower rates of cirrhosis decompensation and HCC development [163]. Also, particular consideration should be given to the potential benefits of antidiabetic drugs such as pioglitazone and sodium-glucose cotransporter 2 (SGLT2) inhibitors, which have been shown to be effective in patients with concurrent T2D and NAFLD [164, 165], although larger RCTs are required. Similarly, the use of the glucagon-like peptide 1 (GLP-1) receptor agonists such as liraglutide or semaglutide is very promising as there is evidence that these agents impact liver histology and can also improve cardiovascular outcomes in patients with T2D [166-169]. Finally, as T2D itself is heterogeneous in its etiology, pathogenesis, and clinical presentation, precision medicine approaches to treat and prevent the disease are being actively investigated but significant barriers to their implementation and research gaps still exist [170].

\section{Individualizing Diet and Exercise Prescription}

As mentioned above, diet and exercise are the cornerstone of NAFLD management. There are various types of diet (e.g., Mediterranean, lowfat diet, and low-carbohydrate diet) as well as different exercise modalities (e.g., high-intensity interval training, moderate-intensity aerobic exercise, and resistance exercise) that have been shown to positively influence NAFLD. Importantly, individualized prescription of diet and exercise therapy may lead to better outcomes.

The goals of dietary intervention should be aligned to patient characteristics and preferences. Weight loss is an important goal in overweight or obese patients as a dose-response relationship exists between the magnitude of weight loss and reduction in liver fat, NASH resolution, and improvement of liver fibrosis $[133,171]$. Thus, a loss of at least $5 \%$ of total body weight has been associated with a decrease in hepatic steatosis, a weight loss of at least 7\% has been shown to lead to NASH resolution, and a weight loss of at least $10 \%$ can result in fibrosis regression or stability [172]. Besides calorie restriction, dietary composition is also an important factor to be considered. Low-carbohydrate diets have shown to be particularly beneficial in patients with atherogenic dyslipidemia and insulin resistance and may favorably impact NAFLD [173]. In particular, the Mediterranean diet, a vegetable-based diet characterized by a high ratio of monounsaturated fatty acids to saturated fatty acids with a total fat accounting for 30-40\% of daily energy consumption, has been shown to have a beneficial effect on glucidic and lipidic metabolism, resulting in improved hepatic steatosis and improved insulin sensitivity in patients with NAFLD [174, 175]. Given that the Mediterranean diet is one of the best studied, current guidelines recommend this particular dietary 
style for NAFLD management [36, 123]. Studies evaluating low-calorie ketogenic diets have shown benefits in terms of reduction of hepatic fat content, but their short duration limits their interpretation. Other approaches such as intermittent fasting regimens hold potential in NAFLD management, but further studies are needed [172]. The science of precision nutrition that considers a holistic approach and allows for the prescription of comprehensive and dynamic nutrition recommendations is beginning to emerge. [176, 177]. Proper consideration of dietary habits, genetic background, health status, microbiome composition, as well as socioeconomics, psychosocial characteristics, and environmental exposures is key for successful intervention.

Exercise determines weight loss-independent benefits in NAFLD at multiple levels [118]. In addition to improving liver histology through several mechanisms [119], it improves cardiorespiratory fitness and may also decrease cardiovascular risk. Importantly, exercise training should be prescribed in a detailed and personalized manner where exercise is considered a "drug" [178]. Clearly, attention should be paid to basal cardiovascular fitness, exercise modality, intensity, frequency, and duration of training. The recent guidelines released by the WHO on sedentary behavior are useful for planning exercise prescription for healthy adults and could, in turn, be used to formulate exercise programs for patients with NAFLD [124]. The specific recommendation is to do at least 150-300 min of moderate-intensity aerobic physical activity or at least 75-150 min of vigorous-intensity aerobic physical activity on a weekly basis, adding muscle strengthening activities involving all major muscle groups 2 days a week [124]. Both aerobic and resistance training improve hepatic fat content and are associated with favorable changes in body composition [179, 180]. Also, high-intensity interval or moderate-intensity continuous aerobic exercise seem to be equally effective with respect to liver fat reduction [181, 182]. Thus, a personalized indication of exercise should consider age, sex, muscle mass, and cardiovascular comorbidities in order to select a safe and effective exercise program.
In recent years, with the emergence of precision exercise medicine [121], the existence of inter-individual differences in the response to a given dose of exercise has also been taken into consideration. However, variables determining such differences remain ill defined and more research assessing physiological responses to interventions in humans is needed [183]. Efforts to find biomarkers to detect those individuals who will benefit more with exercise, such as the META-PREDICT study, have been disappointing so far [184].

Finally, as mentioned above, paying attention to psychosocial factors of any given patient is also relevant as these might influence motivation to both adopt and sustain an exercise program. In this regard, proper support to patients' efforts (i.e., health coaching and motivational interventions) is key to successful adherence to any exercise program aimed at naturalizing physical activity in daily life.

\section{SUMMARY AND OUTLOOK}

The idea of applying precision medicine concepts to NAFLD/NASH management is gaining momentum. As various disease sub-phenotypes with potentially distinct natural history and prognosis and, eventually, different response to therapy, are grouped under the term NAFLD, proper weighing of the differential contribution of different factors to the pathogenesis and clinical expression of NAFLD could help to tailor disease management. Gathering and layering of all pertinent clinical, genetic (i.e., genotyping of major genetic variants or use of PRS), microbiome-related, and cardio-metabolic data should contribute to better defining disease trajectory, risk of progression, and likelihood of response to different therapeutic options in any given patient with NAFLD. Also, in the future, the use of high-throughput omics (i.e., lipidomics proteomics, metabolomics, and glycomics) investigations will help to generate comprehensive biochemical snapshots allowing one to discriminate between patient subgroups $[185,186]$. Also, artificial intelligence tools to integrate and analyze big data as well as to develop algorithms combining the information 

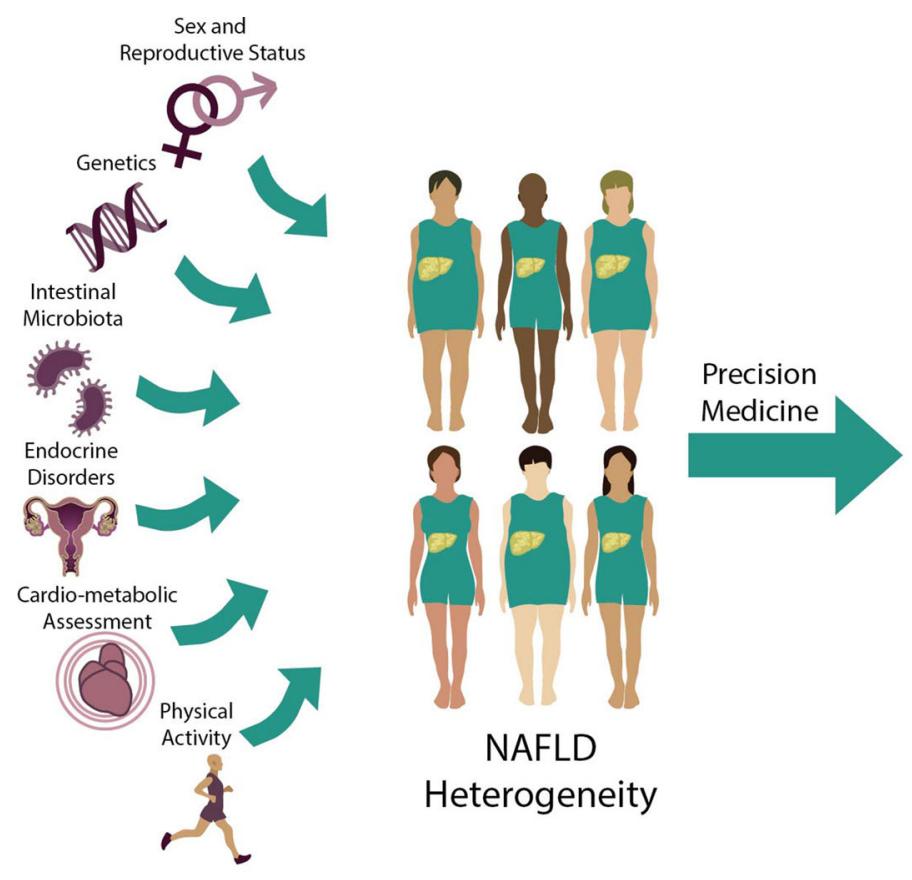

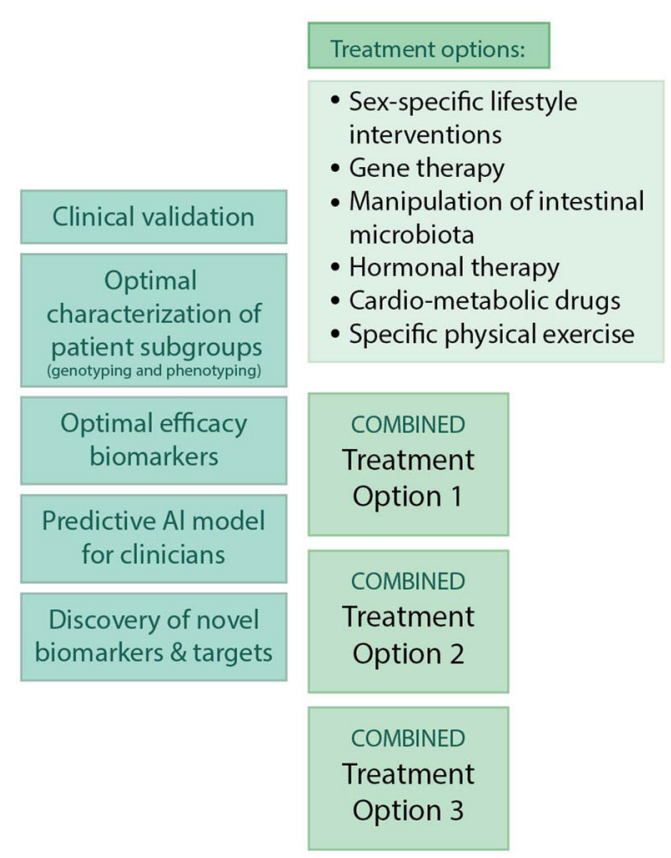

Fig. 1 Precision medicine in NAFLD. Schematic illustration depicting our proposal for a precision medicine approach in NAFLD. Careful consideration of some of the determinants of NAFLD heterogeneity such as sex and reproductive status, genetics, intestinal microbiota diversity, endocrine disorders (such as polycystic ovarian syndrome), cardio-metabolic assessment, and physical activity is suggested in order to develop a more targeted approach based on these factors. An optimal characterization of patient subgroups would allow tailored evaluation and management of patients with NAFLD. To that end, more studies are needed to validate and refine tools such as

through machine learning strategies [187-189] may be of help to better stratifying patients and defining tailored treatment strategies. A more accurate phenotyping of patients may also allow for grouping into more homogenous categories in clinical trials leading to more granular data on the efficacy of drugs in well-defined patient subgroups [26, 190]. Finally, artificial intelligence tools and high-performance computing could be useful to link biological information to health data in electronic medical records, thus advancing the discovery of novel associations using data-mining analysis.

Although significant advances in the path to precision medicine in NAFLD have been made, translation into the clinical arena, bringing microbiota signatures and polygenic risk scores that might be useful for risk stratification and therapeutic decisions. In addition, the use of predictive artificial intelligence (AI) models, discovery and clinical validation of novel biomarkers and targets, and clinical studies that clarify the optimal use of these tools to match specific patient populations with the best treatment options is needed. A multicomponent therapeutic approach might have a better chance of achieving NASH resolution and/or reversal of severe histological outcomes, and of reducing not only liverrelated but also metabolic syndrome-associated morbidity and mortality

recent discoveries to patient management, needs more data and studies to overcome multiple knowledge-based, value-based, and logistic barriers [191]. In the meantime, as practicing clinicians, our efforts should be put into managing NAFLD with an individualized approach. In this regard, adopting the newly proposed nomenclature MAFLD, which uses an inductive approach in contrast to the deductive-negative diagnosis of NAFLD [15, 16], seems logical and has met large consensus [192]. However, additional NAFLD-MAFLD comparative studies are awaited. The positive criteria proposed by an international expert panel separates three subgroups (i.e., obese, lean, and diabetic NAFLD) that may actually prove to 
follow different natural histories [16]. Indeed, a sharper discrimination among subgroups could be possible in the future by applying some of the concepts delineated in the present article (Fig. 1). Identifying more homogenous cohorts of patients with either NAFLD or MAFLD to address natural history and evaluate novel treatment strategies would enlighten our knowledge of the disease and contribute to implementing the practice of precision medicine in NAFLD diagnosis and management.

\section{ACKNOWLEDGEMENTS}

Funding. This work was funded, in part, by grants from the Fondo Nacional De Ciencia y Tecnología de Chile (FONDECYT \#1191145 to M.A.; and \#1200227 to JPA) and the Comisión Nacional de Investigación, Ciencia y Tecnología (CONICYT, AFB170005, CARE, Chile, UC). MA is part of the European-Latin American ESCALON consortium funded by the European Union's Horizon 2020 Research and Innovation Program under grant agreement no. 825510. No other funding or sponsorship was received for this study or publication of this article.

Editorial Assistance. We thank Ms. Valentina Riquelme, Faculty of Arts, Pontificia Universidad Católica, who kindly contributed to this article by drawing Fig. 1 . We are also grateful to Ms. Jacqueline Mole for editing of English.

Authorship. All named authors meet the International Committee of Medical Journal Editors (ICMJE) criteria for authorship for this article, take responsibility for the integrity of the work as a whole, and have given their approval for this version to be published.

Authorship Contributions. AL wrote the first draft of paragraphs 1, 2, and 3. MA and JPA wrote the first draft of paragraph 4 and Tables 1 and 2. Paragraph 5 and Fig. 1. were based on discussion among all the authors. All authors equally participated in editing of the first draft as well as in altering the first submission based on reviewers' comments.

Disclosures. AL is on the editorial board of Advances in Therapy but has nothing else relevant to disclose. JPA and MA have no conflict of interests to disclose with specific reference to the content of the present manuscript.

Compliance with Ethics Guidelines. This article is based on previously conducted studies and does not contain any new studies with human participants or animals performed by any of the authors.

Open Access. This article is licensed under a Creative Commons Attribution-NonCommercial 4.0 International License, which permits any non-commercial use, sharing, adaptation, distribution and reproduction in any medium or format, as long as you give appropriate credit to the original author(s) and the source, provide a link to the Creative Commons licence, and indicate if changes were made. The images or other third party material in this article are included in the article's Creative Commons licence, unless indicated otherwise in a credit line to the material. If material is not included in the article's Creative Commons licence and your intended use is not permitted by statutory regulation or exceeds the permitted use, you will need to obtain permission directly from the copyright holder. To view a copy of this licence, visit http://creativecommons.org/licenses/bync/4.0/.

\section{REFERENCES}

1. Hood L, Friend SH. Predictive, personalized, preventive, participatory (P4) cancer medicine. Nat Rev Clin Oncol. 2011;8(3):184-7.

2. Flores M, Glusman G, Brogaard K, Price ND, Hood L. P4 medicine: how systems medicine will transform the healthcare sector and society. Per Med. 2013;10(6):565-76.

3. Clarke JD, Cherrington NJ. Nonalcoholic steatohepatitis in precision medicine: unraveling the 
factors that contribute to individual variability. Pharmacol Ther. 2015;151:99-106.

4. Johnson KW, Shameer K, Glicksberg BS, et al. Enabling precision cardiology through multiscale biology and systems medicine. JACC Basic Transl Sci. 2017;2(3):311-27.

5. Sookoian S, Pirola CJ. Precision medicine in nonalcoholic fatty liver disease: new therapeutic insights from genetics and systems biology. Clin Mol Hepatol. 2020;26(4):461-75.

6. Carlsson B, Linden D, Brolen G, et al. Review article: the emerging role of genetics in precision medicine for patients with non-alcoholic steatohepatitis. Aliment Pharmacol Ther. 2020;51(12):1305-20.

7. Subramanian M, Wojtusciszyn A, Favre L, et al. Precision medicine in the era of artificial intelligence: implications in chronic disease management. J Transl Med. 2020;18(1):472.

8. Vitale A, Trevisani F, Farinati F, Cillo U. Treatment of hepatocellular carcinoma in the precision medicine era: from treatment stage migration to therapeutic hierarchy. Hepatology. 2020;72(6):2206-18.

9. Lonardo A, Leoni S, Alswat KA, Fouad Y. History of nonalcoholic fatty liver disease. Int $\mathrm{J}$ Mol Sci. 2020;21(16):5888.

10. Cotter TG, Rinella M. Nonalcoholic fatty liver disease 2020: the state of the disease. Gastroenterology. 2020;158(7):1851-64.

11. Younossi ZM, Koenig AB, Abdelatif D, Fazel Y, Henry L, Wymer M. Global epidemiology of nonalcoholic fatty liver disease-meta-analytic assessment of prevalence, incidence, and outcomes. Hepatology. 2016;64(1):73-84.

12. Younossi Z, Tacke F, Arrese M, et al. Global perspectives on nonalcoholic fatty liver disease and nonalcoholic steatohepatitis. Hepatology. 2019;69(6):2672-82.

13. Ballestri S, Mantovani A, Nascimbeni F, Lugari S, Lonardo A. Extra-hepatic manifestations and complications of nonalcoholic fatty liver disease. Future Med Chem. 2019;11(16):2171-92.

14. Fouad Y, Waked I, Bollipo S, Gomaa A, Ajlouni Y, Attia D. What's in a name? Renaming "NAFLD" to "MAFLD." Liver Int. 2020;40(6):1254-61.

15. Eslam M, Sanyal AJ, George J, International Consensus Panel. MAFLD: a consensus-driven proposed nomenclature for metabolic associated fatty liver disease. Gastroenterology. 2020;158(7):1999-2014. e1.
16. Eslam M, Newsome PN, Sarin SK, et al. A new definition for metabolic dysfunction-associated fatty liver disease: an international expert consensus statement. J Hepatol. 2020;73(1):202-9.

17. Mendez-Sanchez N, Arrese M, Gadano A, et al. The Latin American Association for the Study of the Liver (ALEH) position statement on the redefinition of fatty liver disease. Lancet Gastroenterol Hepatol. 2021;6(1):65-72.

18. Shiha G, Alswat K, Al Khatry M, et al. Nomenclature and definition of metabolic-associated fatty liver disease: a consensus from the Middle East and North Africa. Lancet Gastroenterol Hepatol. 2021;6(1):57-64.

19. Shiha G, Korenjak M, Eskridge W, et al. Redefining fatty liver disease: an international patient perspective. Lancet Gastroenterol Hepatol. 2021;6(1): 73-9.

20. Younossi ZM, Rinella ME, Sanyal A, et al. From NAFLD to MAFLD: implications of a premature change in terminology. Hepatology. 2020. https:// doi.org/10.1002/hep.31420.

21. Ratziu V, Rinella M, Beuers U, et al. The times they are a-changin' (for NAFLD as well). J Hepatol. 2020;73(6):1307-9.

22. Cespiati A, Youngson NA, Tourna A, Valenti L. Genetics and epigenetics in the clinic: precision medicine in the management of fatty liver disease. Curr Pharm Des. 2020;26(10):998-1009.

23. Sanyal AJ. Past, present and future perspectives in nonalcoholic fatty liver disease. Nat Rev Gastroenterol Hepatol. 2019;16(6):377-86.

24. Mantovani A, Scorletti E, Mosca A, Alisi A, Byrne CD, Targher G. Complications, morbidity and mortality of nonalcoholic fatty liver disease. Metabolism. 2020;111S:154170.

25. Bellentani S. The epidemiology of non-alcoholic fatty liver disease. Liver Int. 2017;37(Suppl 1):81-4.

26. Ratziu V, Friedman SL. Why do so many NASH trials fail? Gastroenterology. 2020. https://doi.org/10. 1053/j.gastro.2020.05.046.

27. Drenth JPH, Schattenberg JM. The nonalcoholic steatohepatitis (NASH) drug development graveyard: established hurdles and planning for future success. Expert Opin Investig Drugs. 2020;29(12): 1365-75.

28. Arab JP, Arrese M, Trauner M. Recent insights into the pathogenesis of nonalcoholic fatty liver disease. Annu Rev Pathol. 2018;13:321-50. 
29. Suzuki A, Diehl AM. Nonalcoholic steatohepatitis. Annu Rev Med. 2017;68:85-98.

30. Timio M, Di Napoli A, Timio F, Quintaliani G. Does clinical methodology yet exist? G Ital Nefrol. 2016;33(6):gin/33.6.17.

31. Buzzetti E, Pinzani M, Tsochatzis EA. The multiplehit pathogenesis of non-alcoholic fatty liver disease (NAFLD). Metabolism. 2016;65(8):1038-48.

32. Schuster S, Cabrera D, Arrese M, Feldstein AE. Triggering and resolution of inflammation in NASH. Nat Rev Gastroenterol Hepatol. 2018;15(6): 349-64.

33. Vilar-Gomez E, Calzadilla-Bertot L, Wai-Sun Wong $\mathrm{V}$, et al. Fibrosis severity as a determinant of causespecific mortality in patients with advanced nonalcoholic fatty liver disease: a multi-national cohort study. Gastroenterology. 2018;155(2):443-57.e17.

34. Lindenmeyer CC, McCullough AJ. The natural history of nonalcoholic fatty liver disease-an evolving view. Clin Liver Dis. 2018;22(1):11-21.

35. Adams LA, Chan WK. Noninvasive tests in the assessment of NASH and NAFLD fibrosis: now and into the future. Semin Liver Dis. 2020. https://doi. org/10.1055/s-0040-1713006.

36. Chalasani N, Younossi Z, Lavine JE, et al. The diagnosis and management of nonalcoholic fatty liver disease: practice guidance from the American Association for the Study of Liver Diseases. Hepatology. 2018;67(1):328-57.

37. European Association for the Study of the Liver, European Association for the Study of Diabetes, European Association for the Study of Obesity. EASL-EASD-EASO clinical practice guidelines for the management of non-alcoholic fatty liver disease. J Hepatol. 2016;64(6):1388-402.

38. Arab JP, Dirchwolf M, Alvares-da-Silva MR, et al. Latin American Association for the Study of the Liver (ALEH) practice guidance for the diagnosis and treatment of non-alcoholic fatty liver disease. Ann Hepatol. 2020;19(6):674-90.

39. Lonardo A, Lugari S, Nascimbeni F. Non-alcoholic fatty liver disease (NAFLD) diagnosis and management-differentiating the essential from the ancillary and the present from the future. Hepatobiliary Surg Nutr. 2020;9(3):374-8.

40. Tariq R, Axley P, Singal AK. Extra-hepatic manifestations of nonalcoholic fatty liver disease: a review. J Clin Exp Hepatol. 2020;10(1):81-7.

41. Byrne CD, Targher G. NAFLD: a multisystem disease. J Hepatol. 2015;62(1 Suppl):S47-64.
42. Lonardo A, Ballestri S. Perspectives of nonalcoholic fatty liver disease research: a personal point of view. Explor Med. 2020;1:85-107.

43. Lonardo A. Renaming NAFLD to MAFLD: could the LDE system assist in this transition? J Clin Med. 2021;10(3):492.

44. Petta S, Valenti L, Bugianesi E, et al. A "systems medicine" approach to the study of non-alcoholic fatty liver disease. Dig Liver Dis. 2016;48(3):333-42.

45. Ekstedt M, Hagstrom H, Nasr P, et al. Fibrosis stage is the strongest predictor for disease-specific mortality in NAFLD after up to 33 years of follow-up. Hepatology. 2015;61(5):1547-54.

46. Vilar-Gomez E, Chalasani N. Non-invasive assessment of non-alcoholic fatty liver disease: clinical prediction rules and blood-based biomarkers. J Hepatol. 2018;68(2):305-15.

47. Zambrano-Huailla R, Guedes L, Stefano JT, et al. Diagnostic performance of three non-invasive fibrosis scores (Hepamet, FIB-4, NAFLD fibrosis score) in NAFLD patients from a mixed Latin American population. Ann Hepatol. 2020;19(6): 622-6.

48. Ballestri S, Mantovani A, Baldelli E, et al. Liver fibrosis biomarkers accurately exclude advanced fibrosis and are associated with higher cardiovascular risk scores in patients with NAFLD or viral chronic liver disease. Diagnostics (Basel). 2021;11(1):98.

49. Higuera-de-la-Tijera F, Cordova-Gallardo J, Buganza-Torio E, et al. Hepamet fibrosis score in nonalcoholic fatty liver disease patients in Mexico: lower than expected positive predictive value. Dig Dis Sci. 2021. https://doi.org/10.1007/s10620-020-06821-2.

50. Godoy-Matos AF, Silva Junior WS, Valerio CM. NAFLD as a continuum: from obesity to metabolic syndrome and diabetes. Diabetol Metab Syndr. 2020;12:60.

51. Targher G, Byrne CD, Tilg H. NAFLD and increased risk of cardiovascular disease: clinical associations, pathophysiological mechanisms and pharmacological implications. Gut. 2020;69(9):1691-705.

52. Allen AM, Hicks SB, Mara KC, Larson JJ, Therneau TM. The risk of incident extrahepatic cancers is higher in non-alcoholic fatty liver disease than obesity-a longitudinal cohort study. J Hepatol. 2019;71(6):1229-36.

53. Mauvais-Jarvis F, Bairey Merz N, Barnes PJ, et al. Sex and gender: modifiers of health, disease, and medicine. Lancet. 2020;396(10250):565-82. 
54. Gemmati D, Varani K, Bramanti B, et al. "Bridging the gap" everything that could have been avoided if we had applied gender medicine, pharmacogenetics and personalized medicine in the gender-omics and sex-omics era. Int J Mol Sci. 2019;21(1):296.

55. Ballestri S, Nascimbeni F, Baldelli E, Marrazzo A, Romagnoli D, Lonardo A. NAFLD as a sexual dimorphic disease: role of gender and reproductive status in the development and progression of nonalcoholic fatty liver disease and inherent cardiovascular risk. Adv Ther. 2017;34(6):1291-326.

56. Balakrishnan M, Patel P, Dunn-Valadez S, et al. Women have a lower risk of nonalcoholic fatty liver disease but a higher risk of progression vs men: a systematic review and meta-analysis. Clin Gastroenterol Hepatol. 2021;19(1):61-71.e15.

57. Lonardo A, Ballestri S, Chow PKH, Suzuki A. Sex disparity in hepatocellular carcinoma owing to NAFLD and non-NAFLD etiology: epidemiological findings and pathobiological mechanisms. Hepatoma Res. 2020;6:83.

58. Huang DQ, El-Serag HB, Loomba R. Global epidemiology of NAFLD-related HCC: trends, predictions, risk factors and prevention. Nat Rev Gastroenterol Hepatol. 2020. https://doi.org/10. 1038/s41575-020-00381-6.

59. Lonardo A, Nascimbeni F, Ballestri S, et al. Sex differences in nonalcoholic fatty liver disease: state of the art and identification of research gaps. Hepatology. 2019;70(4):1457-69.

60. Venetsanaki V, Polyzos SA. Menopause and nonalcoholic fatty liver disease: a review focusing on therapeutic perspectives. Curr Vasc Pharmacol. 2019;17(6):546-55.

61. Yang JD, Abdelmalek MF, Pang H, et al. Gender and menopause impact severity of fibrosis among patients with nonalcoholic steatohepatitis. Hepatology. 2014;59(4):1406-14.

62. Della TS. Non-alcoholic fatty liver disease as a canonical example of metabolic inflammatorybased liver disease showing a sex-specific prevalence: relevance of estrogen signaling. Front Endocrinol (Lausanne). 2020;11:572490.

63. Lonardo A, Suzuki A. Nonalcoholic fatty liver disease: does sex matter? Hepatobiliary Surg Nutr. 2019;8(2):164-6.

64. Trepo E, Valenti L. Update on NAFLD genetics: from new variants to the clinic. J Hepatol. 2020;72(6): 1196-209.

65. Krawczyk M, Liebe R, Lammert F. Toward genetic prediction of nonalcoholic fatty liver disease trajectories: PNPLA3 and beyond. Gastroenterology. 2020;158(7):1865-80.e1.

66. Sookoian S, Pirola CJ, Valenti L, Davidson NO. Genetic pathways in nonalcoholic fatty liver disease: insights from systems biology. Hepatology. 2020;72(1):330-46.

67. Santos RD, Valenti L, Romeo S. Does nonalcoholic fatty liver disease cause cardiovascular disease? Current knowledge and gaps. Atherosclerosis. 2019;282:110-20.

68. Yki-Jarvinen H, Luukkonen PK. Heterogeneity of non-alcoholic fatty liver disease. Liver Int. 2015;35(12):2498-500.

69. Lonardo A, Ballestri S, Targher G. "Not all forms of NAFLD were created equal". Do metabolic syndrome-related NAFLD and PNPLA3-related NAFLD exert a variable impact on the risk of early carotid atherosclerosis? Atherosclerosis. 2017;257:253-5.

70. Bianco C, Jamialahmadi O, Pelusi S, et al. Non-invasive stratification of hepatocellular carcinoma risk in non-alcoholic fatty liver using polygenic risk scores. J Hepatol. 2020. https://doi.org/10.1016/j. jhep.2020.11.024.

71. Sharpton SR, Schnabl B, Knight R, Loomba R. Current concepts, opportunities, and challenges of gut microbiome-based personalized medicine in nonalcoholic fatty liver disease. Cell Metab. 2021;33(1): 21-32.

72. Houghton D, Stewart CJ, Day CP, Trenell M. Gut microbiota and lifestyle interventions in NAFLD. Int J Mol Sci. 2016;17(4):447.

73. Arab JP, Arrese M, Shah VH. Gut microbiota in nonalcoholic fatty liver disease and alcohol-related liver disease: current concepts and perspectives. Hepatol Res. 2020;50(4):407-18.

74. Bluemel S, Williams B, Knight R, Schnabl B. Precision medicine in alcoholic and nonalcoholic fatty liver disease via modulating the gut microbiota. Am J Physiol Gastrointest Liver Physiol. 2016;311(6): G1018-36.

75. Walker AW, Ince J, Duncan SH, et al. Dominant and diet-responsive groups of bacteria within the human colonic microbiota. ISME J. 2011;5(2): 220-30.

76. Mouzaki M, Loomba R. Insights into the evolving role of the gut microbiome in nonalcoholic fatty liver disease: rationale and prospects for therapeutic intervention. Therap Adv Gastroenterol. 2019;12: 1756284819858470. 
77. Alwahsh SM, Gebhardt R. Dietary fructose as a risk factor for non-alcoholic fatty liver disease (NAFLD). Arch Toxicol. 2017;91(4):1545-63.

78. Jensen T, Abdelmalek MF, Sullivan S, et al. Fructose and sugar: a major mediator of non-alcoholic fatty liver disease. J Hepatol. 2018;68(5):1063-75.

79. Schwabe RF, Greten TF. Gut microbiome in HCC mechanisms, diagnosis and therapy. J Hepatol. 2020;72(2):230-8.

80. Lee G, You HJ, Bajaj JS, et al. Distinct signatures of gut microbiome and metabolites associated with significant fibrosis in non-obese NAFLD. Nat Commun. 2020;11(1):4982.

81. Oh TG, Kim SM, Caussy C, et al. A universal gutmicrobiome-derived signature predicts cirrhosis. Cell Metab. 2020;32(5):878-88.e6.

82. Lonardo A, Carani C, Carulli N, Loria P. "Endocrine NAFLD" a hormonocentric perspective of nonalcoholic fatty liver disease pathogenesis. J Hepatol. 2006;44(6):1196-207.

83. Loria P, Carulli L, Bertolotti M, Lonardo A. Endocrine and liver interaction: the role of endocrine pathways in NASH. Nat Rev Gastroenterol Hepatol. 2009;6(4):236-47.

84. Mantovani A, Nascimbeni F, Lonardo A, et al. Association between primary hypothyroidism and nonalcoholic fatty liver disease: a systematic review and meta-analysis. Thyroid. 2018;28(10):1270-84.

85. Aoki Y, Belin RM, Clickner R, Jeffries R, Phillips L, Mahaffey KR. Serum TSH and total T4 in the United States population and their association with participant characteristics: National Health and Nutrition Examination Survey (NHANES 1999-2002). Thyroid. 2007;17(12):1211-23.

86. Ingoe L, Phipps N, Armstrong G, Rajagopal A, Kamali F, Razvi S. Prevalence of treated hypothyroidism in the community: analysis from general practices in North-East England with implications for the United Kingdom. Clin Endocrinol (Oxf). 2017;87(6):860-4.

87. Lonardo A, Ballestri S, Mantovani A, Nascimbeni F, Lugari S, Targher G. Pathogenesis of hypothyroidism-induced NAFLD: Evidence for a distinct disease entity? Dig Liver Dis. 2019;51(4):462-70.

88. Tanase DM, Gosav EM, Neculae E, et al. Hypothyroidism-induced nonalcoholic fatty liver disease (HIN): mechanisms and emerging therapeutic options. Int J Mol Sci. 2020;21(16):5927.
89. Escobar-Morreale HF. Polycystic ovary syndrome: definition, aetiology, diagnosis and treatment. Nat Rev Endocrinol. 2018;14(5):270-84.

90. Targher G, Rossini M, Lonardo A. Evidence that non-alcoholic fatty liver disease and polycystic ovary syndrome are associated by necessity rather than chance: a novel hepato-ovarian axis? Endocrine. 2016;51(2):211-21.

91. Sarkar M, Terrault N, Chan W, et al. Polycystic ovary syndrome (PCOS) is associated with NASH severity and advanced fibrosis. Liver Int. 2020;40(2): $355-9$.

92. Lonardo A, Mantovani A, Lugari S, Targher G. NAFLD in some common endocrine diseases: prevalence, pathophysiology, and principles of diagnosis and management. Int $\mathrm{J}$ Mol Sci. 2019;20(11):2841.

93. Gariani K, Jornayvaz FR. Pathophysiology of NASH in endocrine diseases. Endocr Connect. 2021. https://doi.org/10.1530/EC-20-0490.

94. Cordoba-Chacon J, Majumdar N, List EO, et al. Growth hormone inhibits hepatic de novo lipogenesis in adult mice. Diabetes. 2015;64(9):3093-103.

95. Xanthakos SA, Crimmins NA, Chernausek SD. Abnormalities in the growth hormone axis and risk of nonalcoholic steatohepatitis: active player or innocent bystander? J Pediatr. 2014;165(1):12-4.

96. Rockall AG, Sohaib SA, Evans D, et al. Hepatic steatosis in Cushing's syndrome: a radiological assessment using computed tomography. Eur J Endocrinol. 2003;149(6):543-8.

97. Tarantino G, Finelli C. Pathogenesis of hepatic steatosis: the link between hypercortisolism and non-alcoholic fatty liver disease. World J Gastroenterol. 2013;19(40):6735-43.

98. Auer MK, Stalla GK, Stieg MR. Investigating the role of cortisol and growth hormone in fatty liver development: fatty liver index in patients with pituitary adenomas. Pituitary. 2016;19(5):461-71.

99. Oikonomou D, Georgiopoulos G, Katsi V, et al. Non-alcoholic fatty liver disease and hypertension: coprevalent or correlated? Eur J Gastroenterol Hepatol. 2018;30(9):979-85.

100. Arrese M, Barrera F, Triantafilo N, Arab JP. Concurrent nonalcoholic fatty liver disease and type 2 diabetes: diagnostic and therapeutic considerations. Expert Rev Gastroenterol Hepatol. 2019;13(9): 849-66.

101. Lonardo A, Lugari S, Ballestri S, Nascimbeni F, Baldelli E, Maurantonio M. A round trip from 
nonalcoholic fatty liver disease to diabetes: molecular targets to the rescue? Acta Diabetol. 2019;56(4): 385-96.

102. Pina AF, Patarrao RS, Ribeiro RT, et al. Metabolic footprint, towards understanding type 2 diabetes beyond glycemia. J Clin Med. 2020;9(8):2588.

103. American Diabetes Association. 2. Classification and diagnosis of diabetes: standards of medical care in diabetes-2021. Diabetes Care. 2021;44(Suppl 1): S15-S33.

104. Amor AJ, Perea V. Dyslipidemia in nonalcoholic fatty liver disease. Curr Opin Endocrinol Diabetes Obes. 2019;26(2):103-8.

105. Schult A, Eriksson H, Wallerstedt S, Kaczynski J. Overweight and hypertriglyceridemia are risk factors for liver cirrhosis in middle-aged Swedish men. Scand J Gastroenterol. 2011;46(6):738-44.

106. Siddiqui MS, Fuchs M, Idowu MO, et al. Severity of nonalcoholic fatty liver disease and progression to cirrhosis are associated with atherogenic lipoprotein profile. Clin Gastroenterol Hepatol. 2015;13(5): 1000-8.e3.

107. Arguello G, Balboa E, Arrese M, Zanlungo S. Recent insights on the role of cholesterol in non-alcoholic fatty liver disease. Biochim Biophys Acta. 2015;1852(9):1765-78.

108. Ioannou GN. The role of cholesterol in the pathogenesis of NASH. Trends Endocrinol Metab. 2016;27(2):84-95.

109. Lonardo A, Loria P. Potential for statins in the chemoprevention and management of hepatocellular carcinoma. J Gastroenterol Hepatol. 2012;27(11):1654-64.

110. Vargas JI, Arrese M, Shah VH, Arab JP. Use of statins in patients with chronic liver disease and cirrhosis: current views and prospects. Curr Gastroenterol Rep. 2017;19(9):43.

111. Nascimbeni F, Pellegrini E, Lugari S, et al. Statins and nonalcoholic fatty liver disease in the era of precision medicine: more friends than foes. Atherosclerosis. 2019;284:66-74.

112. Ballestri S, Capitelli M, Fontana MC, et al. Direct oral anticoagulants in patients with liver disease in the era of non-alcoholic fatty liver disease global epidemic: a narrative review. Adv Ther. 2020;37(5): 1910-32.

113. Dongiovanni P, Paolini E, Corsini A, Sirtori CR, Ruscica M. NAFLD or MAFLD diagnoses and cardiovascular diseases: from epidemiology to drug approaches. Eur J Clin Invest. 2021:e13519.
114. Adams LA, Crawford DH, Stuart K, et al. The impact of phlebotomy in nonalcoholic fatty liver disease: a prospective, randomized, controlled trial. Hepatology. 2015;61(5):1555-64.

115. Britton L, Bridle K, Reiling J, et al. Hepatic iron concentration correlates with insulin sensitivity in nonalcoholic fatty liver disease. Hepatol Commun. 2018;2(6):644-53.

116. Morisco F, Pagliaro L, Caporaso N, et al. Consensus recommendations for managing asymptomatic persistent non-virus non-alcohol related elevation of aminotransferase levels: suggestions for diagnostic procedures and monitoring. Dig Liver Dis. 2008;40(7):585-98.

117. Gerber L, Otgonsuren M, Mishra A, et al. Non-alcoholic fatty liver disease (NAFLD) is associated with low level of physical activity: a population-based study. Aliment Pharmacol Ther. 2012;36(8):772-81.

118. Romero-Gomez M, Zelber-Sagi S, Trenell M. Treatment of NAFLD with diet, physical activity and exercise. J Hepatol. 2017;67(4):829-46.

119. Thorp A, Stine JG. Exercise as medicine: the impact of exercise training on nonalcoholic fatty liver disease. Curr Hepatol Rep. 2020;19:402-11.

120. Su CL, Wang L, Ho CC, et al. Physical activity is associated with lower health care costs among Taiwanese individuals with diabetes mellitus. Medicine (Baltimore). 2020;99(14):e19613.

121. Ross R, Goodpaster BH, Koch LG, et al. Precision exercise medicine: understanding exercise response variability. Br J Sports Med. 2019;53(18):1141-53.

122. Ramirez-Velez R, Izquierdo M. Editorial: Precision physical activity and exercise prescriptions for disease prevention: the effect of interindividual variability under different training approaches. Front Physiol. 2019;10:646.

123. Younossi ZM, Corey KE, Lim JK. AGA clinical practice update on lifestyle modification using diet and exercise to achieve weight loss in the management of nonalcoholic fatty liver disease: expert review. Gastroenterology. 2021;160(3):912-8.

124. WHO. WHO guidelines on physical activity and sedentary behaviour. Geneva: World Health Organization; 2020. 2020.

125. Chen Z, Yu Y, Cai J, Li H. Emerging molecular targets for treatment of nonalcoholic fatty liver disease. Trends Endocrinol Metab. 2019;30(12):903-14.

126. Horn CL, Ta AC, Gunn NT. Current and emerging treatments for non-alcoholic steatohepatitis. Curr Hepatology Rep. 2020;19:391-401. 
127. Lonardo A, Suzuki A. Sexual dimorphism of NAFLD in adults. Focus on clinical aspects and implications for practice and translational research. J Clin Med. 2020;9(5):1278.

128. Kane AE, Sinclair DA, Mitchell JR, Mitchell SJ. Sex differences in the response to dietary restriction in rodents. Curr Opin Physiol. 2018;6:28-34.

129. Austad SN, Fischer KE. Sex differences in lifespan. Cell Metab. 2016;23(6):1022-33.

130. Mattison JA, Colman RJ, Beasley TM, et al. Caloric restriction improves health and survival of rhesus monkeys. Nat Commun. 2017;8:14063.

131. Brennan L, Gibbons H. Sex matters: a focus on the impact of biological sex on metabolomic profiles and dietary interventions. Proc Nutr Soc. 2020;79(2):205-9.

132. Christensen P, Meinert Larsen T, Westerterp-Plantenga $\mathrm{M}$, et al. Men and women respond differently to rapid weight loss: metabolic outcomes of a multicentre intervention study after a low-energy diet in 2500 overweight, individuals with pre-diabetes (PREVIEW). Diabetes Obes Metab. 2018;20(12): 2840-51.

133. Vilar-Gomez E, Martinez-Perez Y, Calzadilla-Bertot $\mathrm{L}$, et al. Weight loss through lifestyle modification significantly reduces features of nonalcoholic steatohepatitis. Gastroenterology. 2015;149(2): 367-78 e5 (quiz e14-5).

134. DiStefano JK. NAFLD and NASH in postmenopausal women: implications for diagnosis and treatment. Endocrinology. 2020;161(10):bqaa134.

135. Thomson ZO, Kelly JT, Sainsbury A, Reeves MM. Weight loss outcomes in premenopausal versus postmenopausal women during behavioral weight loss interventions: a systematic review and metaanalysis. Menopause. 2020;28(3):337-46.

136. Romeo S, Sanyal A, Valenti L. Leveraging human genetics to identify potential new treatments for fatty liver disease. Cell Metab. 2020;31(1):35-45.

137. Shen J, Wong GL, Chan HL, et al. PNPLA3 gene polymorphism and response to lifestyle modification in patients with nonalcoholic fatty liver disease. J Gastroenterol Hepatol. 2015;30(1):139-46.

138. Krawczyk M, Jimenez-Aguero R, Alustiza JM, et al. PNPLA3 p.I148M variant is associated with greater reduction of liver fat content after bariatric surgery. Surg Obes Relat Dis. 2016;12(10):1838-46.

139. Luukkonen PK, Qadri S, Lehtimaki TE, et al. The PNPLA3-I148M variant confers an antiatherogenic lipid profile in insulin-resistant patients. J Clin Endocrinol Metab. 2021;106(1):e300-15.

140. Linden D, Ahnmark A, Pingitore P, et al. Pnpla3 silencing with antisense oligonucleotides ameliorates nonalcoholic steatohepatitis and fibrosis in Pnpla3 I148M knock-in mice. Mol Metab. 2019;22: 49-61.

141. Lang S, Schnabl B. Microbiota and fatty liver disease-the known, the unknown, and the future. Cell Host Microbe. 2020;28(2):233-44.

142. Kwong EK, Puri P. Gut microbiome changes in nonalcoholic fatty liver disease \& alcoholic liver disease. Transl Gastroenterol Hepatol. 2021;6:3.

143. Chu H, Duan Y, Yang L, Schnabl B. Small metabolites, possible big changes: a microbiota-centered view of non-alcoholic fatty liver disease. Gut. 2019;68(2):359-70.

144. Sharpton SR, Maraj B, Harding-Theobald E, Vittinghoff E, Terrault NA. Gut microbiome-targeted therapies in nonalcoholic fatty liver disease: a systematic review, meta-analysis, and meta-regression. Am J Clin Nutr. 2019;110(1):139-49.

145. Maldonado-Gomez MX, Martinez I, Bottacini F, et al. Stable engraftment of Bifidobacterium longum AH1206 in the human gut depends on individualized features of the resident microbiome. Cell Host Microbe. 2016;20(4):515-26.

146. Liu L, Yu Y, Zhao M, et al. Benefits of levothyroxine replacement therapy on nonalcoholic fatty liver disease in subclinical hypothyroidism patients. Int J Endocrinol. 2017;2017:5753039.

147. Lugari S, Mantovani A, Nascimbeni F, Lonardo A. Hypothyroidism and nonalcoholic fatty liver disease - a chance association? Horm Mol Biol Clin Investig. 2018;41(1). https://doi.org/10.1515/ hmbci-2018-0047.

148. Sinha RA, Bruinstroop E, Singh BK, Yen PM. Thyroid hormones and thyromimetics: a new approach to nonalcoholic steatohepatitis? Hepatology. 2020;72(2):770-1.

149. Harrison SA, Bashir MR, Guy CD, et al. Resmetirom (MGL-3196) for the treatment of non-alcoholic steatohepatitis: a multicentre, randomised, doubleblind, placebo-controlled, phase 2 trial. Lancet. 2019;394(10213):2012-24.

150. Maseroli E, Comeglio P, Corno C, et al. Testosterone treatment is associated with reduced adipose tissue dysfunction and nonalcoholic fatty liver disease in obese hypogonadal men. J Endocrinol Invest. 2020. https://doi.org/10.1007/s40618-020-01381-8. 
151. Yassin AA, Alwani M, Talib R, et al. Long-term testosterone therapy improves liver parameters and steatosis in hypogonadal men: a prospective controlled registry study. Aging Male. 2021:1-11. https://doi.org/10.1080/13685538.2020.1867094.

152. Takahashi Y. The role of growth hormone and insulin-like growth factor-I in the liver. Int J Mol Sci. 2017;18(7):1447.

153. Moolla A, Motohashi K, Marjot T, et al. A multidisciplinary approach to the management of NAFLD is associated with improvement in markers of liver and cardio-metabolic health. Frontline Gastroenterol. 2019;10(4):337-46.

154. Editorial. Precision cardiology in the digital era. Nat Rev Cardiol. 2019;16(11):637.

155. Corral-Acero J, Margara F, Marciniak M, et al. The "digital twin" to enable the vision of precision cardiology. Eur Heart J. 2020;41(48):4556-64.

156. Targher G, Corey KE, Byrne CD. NAFLD, and cardiovascular and cardiac diseases: factors influencing risk, prediction and treatment. Diabetes Metab. 2020;47(2):101215.

157. Leopold JA, Loscalzo J. Emerging role of precision medicine in cardiovascular disease. Circ Res. 2018;122(9):1302-15.

158. Su X, Cheng Y, Chang D. Lipid-lowering therapy: guidelines to precision medicine. Clin Chim Acta. 2020;514:66-73.

159. Mantovani A, Petracca G, Beatrice G, Tilg H, Byrne CD, Targher G. Non-alcoholic fatty liver disease and risk of incident diabetes mellitus: an updated metaanalysis of 501022 adult individuals. Gut. 2020. https://doi.org/10.1136/gutjnl-2020-322572.

160. American Diabetes Association. 3. Prevention or delay of type 2 diabetes: standards of medical care in diabetes-2021. Diabetes Care. 2021;44(Suppl 1): S34-S9.

161. Ampuero J, Aller R, Gallego-Duran R, et al. Significant fibrosis predicts new-onset diabetes mellitus and arterial hypertension in patients with NASH. J Hepatol. 2020;73(1):17-25.

162. Introduction: Standards of Medical Care in Diabetes-2021. Diabetes Care. 2021;44(Suppl 1): S1-S2.

163. Vilar-Gomez E, Calzadilla-Bertot L, Wong VW, et al. Type 2 diabetes and metformin use associate with outcomes of patients with nonalcoholic steatohepatitis-related, Child-Pugh A cirrhosis. Clin Gastroenterol Hepatol. 2021;19(1):136-45.e6.
164. Xing B, Zhao Y, Dong B, Zhou Y, Lv W, Zhao W. Effects of sodium-glucose cotransporter 2 inhibitors on non-alcoholic fatty liver disease in patients with type 2 diabetes: a meta-analysis of randomized controlled trials. J Diabetes Investig. 2020;11(5): 1238-47.

165. Blazina I, Selph S. Diabetes drugs for nonalcoholic fatty liver disease: a systematic review. Syst Rev. 2019;8(1):295.

166. Lv X, Dong Y, Hu L, Lu F, Zhou C, Qin S. Glucagonlike peptide-1 receptor agonists (GLP-1 RAs) for the management of nonalcoholic fatty liver disease (NAFLD): A systematic review. Endocrinol Diabetes Metab. 2020;3(3):e00163.

167. Mantovani A, Byrne CD, Scorletti E, Mantzoros CS, Targher G. Efficacy and safety of anti-hyperglycaemic drugs in patients with non-alcoholic fatty liver disease with or without diabetes: An updated systematic review of randomized controlled trials. Diabetes Metab. 2020;46(6):427-41.

168. Mantovani A, Petracca G, Beatrice G, et al. Glucagon-like peptide-1 receptor agonists for treatment of nonalcoholic fatty liver disease and nonalcoholic steatohepatitis: an updated meta-analysis of randomized controlled trials. Metabolites. 2021;11(2): 73. https://doi.org/10.3390/metabo11020073.

169. Dai Y, He H, Li S, Yang L, Wang X, Liu Z, Zhenmei A. Comparison of the efficacy of glucagon-like peptide- 1 receptor agonists in patients with metabolic associated fatty liver disease: updated systematic review and meta-analysis. Front Endocrinol (Lausanne). 2021;11:622589. https://doi.org/10. $3389 /$ fendo.2020.622589.

170. Chung WK, Erion K, Florez JC, et al. Precision medicine in diabetes: a consensus report from the American Diabetes Association (ADA) and the European Association for the Study of Diabetes (EASD). Diabetologia. 2020;63(9):1671-93.

171. Younossi ZM, Corey KE, Lim JK. AGA Clinical practice update on lifestyle modification using diet and exercise to achieve weight loss in the management of nonalcoholic fatty liver disease (NAFLD): expert review. Gastroenterology. 2021;160(3): 912-8.

172. Viveiros K. The role of life style modifications in comprehensive non-alcoholic fatty liver disease treatment. Clin Liver Dis (Hoboken). 2021;17(1): $11-4$.

173. Noakes TD, Windt J. Evidence that supports the prescription of low-carbohydrate high-fat diets: a narrative review. Br J Sports Med. 2017;51(2):133-9. 
174. Plaz Torres MC, Aghemo A, Lleo A, et al. Mediterranean diet and NAFLD: what we know and questions that still need to be answered. Nutrients. 2019;11(12):2971.

175. Katsagoni CN, Georgoulis M, Papatheodoridis GV, Panagiotakos DB, Kontogianni MD. Effects of lifestyle interventions on clinical characteristics of patients with non-alcoholic fatty liver disease: a meta-analysis. Metabolism. 2017;68:119-32.

176. Simopoulos AP, Serhan CN, Bazinet RP. The need for precision nutrition, genetic variation and resolution in Covid-19 patients. Mol Aspects Med. 2021: 100943.

177. O'Grady J, Shanahan F. Macronutrients, microbiome and precision nutrition. Curr Opin Gastroenterol. 2021;37(2):145-51.

178. Sallis R. Exercise is medicine: a call to action for physicians to assess and prescribe exercise. Phys Sportsmed. 2015;43(1):22-6.

179. Zelber-Sagi S, Buch A, Yeshua H, et al. Effect of resistance training on non-alcoholic fatty-liver disease a randomized-clinical trial. World J Gastroenterol. 2014;20(15):4382-92.

180. Hashida R, Kawaguchi T, Bekki M, et al. Aerobic vs. resistance exercise in non-alcoholic fatty liver disease: a systematic review. J Hepatol. 2017;66(1): 142-52.

181. Abdelbasset WK, Tantawy SA, Kamel DM, et al. Effects of high-intensity interval and moderate-intensity continuous aerobic exercise on diabetic obese patients with nonalcoholic fatty liver disease: a comparative randomized controlled trial. Medicine (Baltimore). 2020;99(10):e19471.

182. Zhang HJ, He J, Pan LL, et al. Effects of moderate and vigorous exercise on nonalcoholic fatty liver disease: a randomized clinical trial. JAMA Intern Med. 2016;176(8):1074-82.

183. Ansdell P, Thomas K, Hicks KM, Hunter SK, Howatson G, Goodall S. Physiological sex differences affect the integrative response to exercise: acute and chronic implications. Exp Physiol. 2020;105(12):2007-21.

184. CORDIS EC. Final report summary-META-PREDICT (developing predictors of the health benefits of exercise for individuals). 2016. https://cordis. europa.eu/project/id/277936/reporting. Accessed 2 Feb 2021

185. Pirola CJ, Sookoian S. Multiomics biomarkers for the prediction of nonalcoholic fatty liver disease severity. World J Gastroenterol. 2018;24(15): 1601-15.
186. Perakakis N, Stefanakis K, Mantzoros CS. The role of omics in the pathophysiology, diagnosis and treatment of non-alcoholic fatty liver disease. Metabolism. 2020;111S:154320.

187. Goldman O, Ben-Assuli O, Rogowski O, et al. Nonalcoholic fatty liver and liver fibrosis predictive analytics: risk prediction and machine learning techniques for improved preventive medicine. J Med Syst. 2021;45(2):22.

188. Ahn JC, Connell A, Simonetto DA, Hughes C, Shah $\mathrm{VH}$. The application of artificial intelligence for the diagnosis and treatment of liver diseases. Hepatology. 2020. https://doi.org/10.1002/hep.31603.

189. Perakakis N, Yazdani A, Karniadakis GE, Mantzoros C. Omics, big data and machine learning as tools to propel understanding of biological mechanisms and to discover novel diagnostics and therapeutics. Metabolism. 2018;87:A1-9.

190. Ampuero J, Romero-Gomez M. Stratification of patients in NASH clinical trials: a pitfall for trial success. JHEP Rep. 2020;2(5):100148.

191. Whitcomb DC. Primer on precision medicine for complex chronic disorders. Clin Transl Gastroenterol. 2019;10(7):e00067.

192. Bianco C, Romeo S, Petta S, Long MT, Valenti L. MAFLD vs NAFLD: let the contest begin! Liver Int. 2020;40(9):2079-81.

193. Lin ZH, Xin YN, Dong QJ, et al. Performance of the aspartate aminotransferase-to-platelet ratio index for the staging of hepatitis C-related fibrosis: an updated meta-analysis. Hepatology. 2011;53(3): 726-36.

194. Lee J, Vali Y, Boursier J, et al. Prognostic accuracy of FIB-4, NAFLD fibrosis score, and APRI for NAFLDrelated events: a systematic review. Liver Int. 2021;41(2):261-70.

195. Sterling RK, Lissen E, Clumeck N, et al. Development of a simple noninvasive index to predict significant fibrosis in patients with $\mathrm{HIV} / \mathrm{HCV}$ coinfection. Hepatology. 2006;43(6):1317-25.

196. Wu Y, Kumar R, Huang J, Wang M, Zhu Y, Lin S. FIB-4 cut-off should be re-evaluated in patients with metabolic associated fatty liver disease (MAFLD). J Hepatol. 2021;74(1):247-8.

197. Angulo P, Hui JM, Marchesini G, et al. The NAFLD fibrosis score: a noninvasive system that identifies liver fibrosis in patients with NAFLD. Hepatology. $2007 ; 45(4): 846-54$.

198. Ampuero J, Pais R, Aller R, et al. Development and validation of Hepamet fibrosis scoring system-a 
simple, noninvasive test to identify patients with nonalcoholic fatty liver disease with advanced fibrosis. Clin Gastroenterol Hepatol. 2020;18(1): 216-25.e5.

199. Poynard T, Munteanu M, Deckmyn O, et al. Validation of liver fibrosis biomarker (FibroTest) for assessing liver fibrosis progression: proof of concept and first application in a large population. J Hepatol. 2012;57(3):541-8.

200. Ratziu V, Massard J, Charlotte F, et al. Diagnostic value of biochemical markers (FibroTest-FibroSURE) for the prediction of liver fibrosis in patients with non-alcoholic fatty liver disease. BMC Gastroenterol. 2006;6:6.

201. Loomba R, Adams LA. Advances in non-invasive assessment of hepatic fibrosis. Gut. 2020;69(7): 1343-52.

202. Guajardo-Salinas GE, Hilmy A. Prevalence of nonalcoholic fatty liver disease (NAFLD) and utility of FIBROspect II to detect liver fibrosis in morbidly obese Hispano-American patients undergoing gastric bypass. Obes Surg. 2010;20(12):1647-53.

203. Aykut UE, Akyuz U, Yesil A, et al. A comparison of FibroMeter NAFLD Score, NAFLD fibrosis score, and transient elastography as noninvasive diagnostic tools for hepatic fibrosis in patients with biopsyproven non-alcoholic fatty liver disease. Scand J Gastroenterol. 2014;49(11):1343-8.

204. Vali Y, Lee J, Boursier J, et al. Enhanced liver fibrosis test for the non-invasive diagnosis of fibrosis in patients with NAFLD: a systematic review and metaanalysis. J Hepatol. 2020;73(2):252-62.

205. Harrison SA, Ratziu V, Boursier J, et al. A bloodbased biomarker panel (NIS4) for non-invasive diagnosis of non-alcoholic steatohepatitis and liver fibrosis: a prospective derivation and global validation study. Lancet Gastroenterol Hepatol. 2020;5(11):970-85.

206. Siddiqui MS, Vuppalanchi R, Van Natta ML, et al. Vibration-controlled transient elastography to assess fibrosis and steatosis in patients with nonalcoholic fatty liver disease. Clin Gastroenterol Hepatol. 2019;17(1):156-63.e2.

207. Lee DH, Cho EJ, Bae JS, et al. Accuracy of two-dimensional shear wave elastography and attenuation imaging for evaluation of patients with nonalcoholic steatohepatitis. Clin Gastroenterol Hepatol. 2020. https://doi.org/10.1016/j.cgh.2020.05.034.

208. Liu H, Fu J, Hong R, Liu L, Li F. Acoustic radiation force impulse elastography for the non-invasive evaluation of hepatic fibrosis in non-alcoholic fatty liver disease patients: a systematic review \& metaanalysis. PLoS One. 2015;10(7):e0127782.

209. Singh S, Venkatesh SK, Loomba R, et al. Magnetic resonance elastography for staging liver fibrosis in non-alcoholic fatty liver disease: a diagnostic accuracy systematic review and individual participant data pooled analysis. Eur Radiol. 2016;26(5): 1431-40. 